\title{
In-beam spectroscopy of heavy elements
}

\author{
Ch. Theisen ${ }^{\mathrm{a}}$, P.T. Greenlees ${ }^{\mathrm{b}}$, T.-L. Khoo ${ }^{\mathrm{c}}$, P. Chowdhury $^{\mathrm{d}}$, T. Ishii ${ }^{\mathrm{e}}$ \\ ${ }^{a}$ CEA, Centre de Saclay, IRFU/Service de Physique Nucléaire, F-91191 Gif-sur-Yvette, \\ France \\ ${ }^{b}$ University of Jyväskylä, Department of Physics, P.O. Box 35, FI-40014 Jyväskylä, \\ Finland \\ ${ }^{c}$ Argonne National Laboratory, Argonne, Illinois 60439, USA \\ ${ }^{d}$ University of Massachusetts Lowell, Lowell, Massachusetts 01854, USA \\ ${ }^{e}$ Advanced Science Research Center, Japan Atomic Energy Agency, Tokai, Ibaraki \\ 319-1195, Japan
}

\begin{abstract}
Traditionally the experimental study of heavy and superheavy elements has belonged to the realm of decay spectroscopy and nuclear reactions. Only in the past twenty years or so has it become feasible to study nuclei with $Z=96$ and beyond with in-beam spectroscopic techniques. Since the pioneering studies in the late 1990s, development of both instrumentation and experimental techniques has resulted in a significant lowering of the spectroscopic limit for in-beam measurements. Such measurements give access to a wide range of nuclear structure observables which in general are beyond the reach of other techniques. The current review aims to present the most recent developments and results in the field, building upon previous reviews with a similar theme.
\end{abstract}

Keywords: In-beam spectroscopy, heavy elements, superheavy elements, nuclear structure, isomeric states

\section{Introduction}

The past two decades have witnessed steady progress in the development of in-beam spectroscopic techniques. The lowering of spectroscopic limits to unprecedented levels and the selectivity provided by modern techniques have allowed in-beam studies to be employed in a wider range of heavier nuclei, yielding a wealth of new experimental data. These data have been effectively 
applied to test modern nuclear structure theories, leading to the identification of deficiencies which are currently being addressed by the theoretical community and which are briefly discussed in the current manuscript. As is the case with many of the experimental and theoretical studies presented in this volume, the main theme behind in-beam spectroscopic studies is to further our understanding of the spherical superheavy island of stability. It is well-known that the different theoretical models give differing results concerning the properties of nuclei in and around the island of stability, and also on the location and extent of the island (for details see the theoretical reviews in this volume). At least in part, these differences in the predictions can be traced back to the ordering and energies of the underlying single-particle levels. An experimental determination of the structure and configurations of excited states in heavy nuclei can therefore have a direct impact on this discussion.

Historically, the experimental study of heavy elements has been approached through decay spectroscopy and a limited number of transfer reaction experiments. Such approaches generally only give information on a limited number of nuclear states, and are subject to the rather selective nature of the decay processes and reaction mechanisms. The field of decay spectroscopy has also benefitted in recent years from technological developments and multi-detector systems, resulting in much more detailed spectroscopy and the possibility of putting level assignments on a firmer basis through determination of transition multipolarities. The current status of the field of decay spectroscopy is reviewed elsewhere in this volume. The application of in-beam techniques to heavy nuclei can give access to a range of experimental observables which complement beautifully the information from decay spectroscopic studies. It is now possible to make systematic studies of rotational bands and moments of inertia in a reasonably wide range of nuclei (spanning ranges in both $N$ and $Z$ ), which in turn provide information on the development of deformation, collectivity and pairing. At higher spins, rotational alignment properties and blocking arguments can give insight into the particles active at the Fermi surface. In particular, the high- $j$ orbitals which are most sensitive to rotational alignment effects through the Coriolis interaction are also those which effectively form the boundary to the island of stability. Here again, confrontation of theoretical models with experimental data on the location of the high- $j$ states can impact the discussion of the properties of SuperHeavy Elements (SHE).

While most of the experiments discussed in the review were made using 
fusion-evaporation reactions, the use of transfer reactions, either of a few particles, or of a large number of particles in a deep-inelastic collision, is still a very powerful tool in the study of heavy nuclei. In-beam experiments generally rely on discrete-line spectroscopy, but another approach is to measure the total energy and multiplicity of gamma-rays emitted in the decay of particular nucleus. This "continuum" approach allows the fission barrier height to be determined, and provides information on the reaction mechanism.

The following article aims to review recent progress in in-beam spectroscopic studies of heavy nuclei. As the field was reviewed as recently as 2008 [1], emphasis is placed on developments since that time, even though some overlap is inevitable. The next section (2) describes the current status of experimental apparatus and techniques, followed by sections dealing with even-even (3) and odd-mass nuclei (4), two-quasiparticle states (5), discussion of the experimental findings and comparison with theory (6) and finally ending with an evaluation of the perspectives for further progress in the field (7).

The current status of experimental knowledge on nuclei from $\mathrm{Cm}$ to $\mathrm{Db}$ is summarised in Fig. 1, which shows the ground-state spin assignments along with the number of known excited states which are classified with a colour code. The figure is an updated version of that presented in the review of Herzberg and Greenlees [1], which is intended to provide insight into the progress made since late 2007 . Table 1 lists the in-beam studies performed in the same region.

Table 1: In-beam studies performed in the $\mathrm{Cm}$ to $\mathrm{Db}$ region.

\begin{tabular}{lll}
\hline Nucleus & References & Notes \\
\hline \hline${ }^{242} \mathrm{Cm}$ & Abu Saleem et al. [2] & Transfer \\
\hline${ }^{246} \mathrm{Cm}$ & Abu Saleem [3] & Transfer \\
\hline${ }^{247} \mathrm{Cm}$ & Hota et al. [4] & Transfer \\
\hline${ }^{248} \mathrm{Cm}$ & Hackman et al. [5] & Coulomb excitation \\
& Abu Saleem [3] & Inelastic scattering \\
\hline${ }^{249} \mathrm{Cm}$ & Ishii et al. [6] & Transfer \\
& Tandel et al. [7] & Transfer \\
\hline${ }^{250} \mathrm{Cm}$ & Ishii et al. [8] & Transfer \\
& Hota [9] & Transfer \\
\hline${ }^{248} \mathrm{Cf}$ & Takahashi et al. [10] & Transfer \\
\hline
\end{tabular}


Table 1: (continued)

\begin{tabular}{|c|c|c|}
\hline Nucleus & References & Notes \\
\hline \multirow{2}{*}{${ }^{249} \mathrm{Cf}$} & Tandel et al. [7] & Inelastic scattering \\
\hline & Hota et al. 4 & Inelastic scattering \\
\hline \multirow{2}{*}{${ }^{250} \mathrm{Cf}$} & Takahashi et al. [10] & Transfer \\
\hline & Hota et al. 4. & Transfer \\
\hline${ }^{251} \mathrm{Cf}$ & Qiu et al. [11] & Inelastic scattering \\
\hline${ }^{252} \mathrm{Cf}$ & Takahashi et al. 10] & Transfer \\
\hline${ }^{246} \mathrm{Fm}$ & Piot et al. $[12$ & \\
\hline${ }^{248} \mathrm{Fm}$ & Ketelhut [13] & \\
\hline \multirow{2}{*}{${ }^{250} \mathrm{Fm}$} & Bastin et al. 14] & \\
\hline & Greenlees et al. 15 & High- $K$ band \\
\hline${ }^{251} \mathrm{Md}$ & Chatillon et al. [16] & \\
\hline \multirow{3}{*}{${ }^{252} \mathrm{No}$} & Herzberg et al. [17] & \\
\hline & Leppänen et al. 18 & Recoil Fission Tagging \\
\hline & Sulignano et al. [19] & High- $K$ band \\
\hline \multirow{3}{*}{${ }^{253} \mathrm{No}$} & Herzberg et al. [20] & Electron spectroscopy \\
\hline & Reiter et al. 21] & \\
\hline & Herzberg et al. [22] & \\
\hline \multirow{8}{*}{${ }^{254} \mathrm{No}$} & Reiter et al. [23] & \\
\hline & Leino et al. [24] & \\
\hline & Reiter et al. 25] & Entry distribution \\
\hline & Butler et al. 26] & Electron spectroscopy \\
\hline & Herzberg et al. 20 & \\
\hline & Humphreys et al. 27 & Electron spectroscopy \\
\hline & Eeckhaudt et al. [28] & \\
\hline & Henning et al. 29] & Fission Barrier \\
\hline${ }^{255} \mathrm{Lr}$ & Ketelhut et al. [30] & \\
\hline${ }^{256} \mathrm{Rf}$ & Greenlees et al. 31] & \\
\hline
\end{tabular}

\section{Experimental Techniques}

The steady progress in in-beam studies of the heaviest nuclei is mainly rooted in the exploitation and further development of the recoil-decay tagging technique, which was first employed in the 1980s and 1990s [32, 33]: see Fig. 2 , Fusion-evaporation reactions are used to produce the nuclei of interest with 


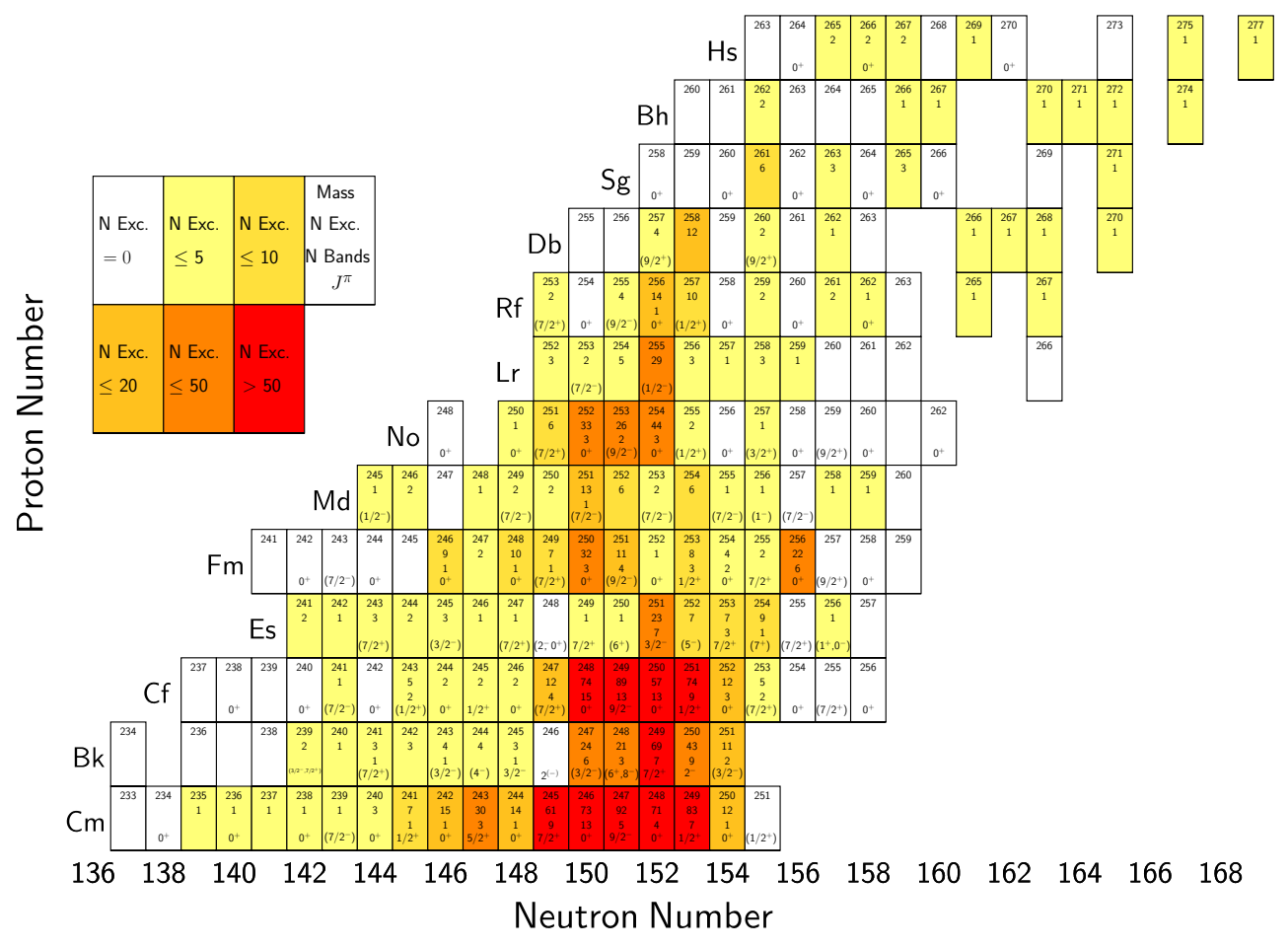

Figure 1: (Color online) Summary of the experimental data available on nuclei from $\mathrm{Cm}$ to $\mathrm{Db}$. The mass number, the number of known excited levels, the number of assigned rotational bands and the ground state spin are given for each isotope. The colour code gives an impression of the level knowledge for a particular isotope. The figure is an updated version of Fig. 1 from the review of Herzberg and Greenlees [1. 


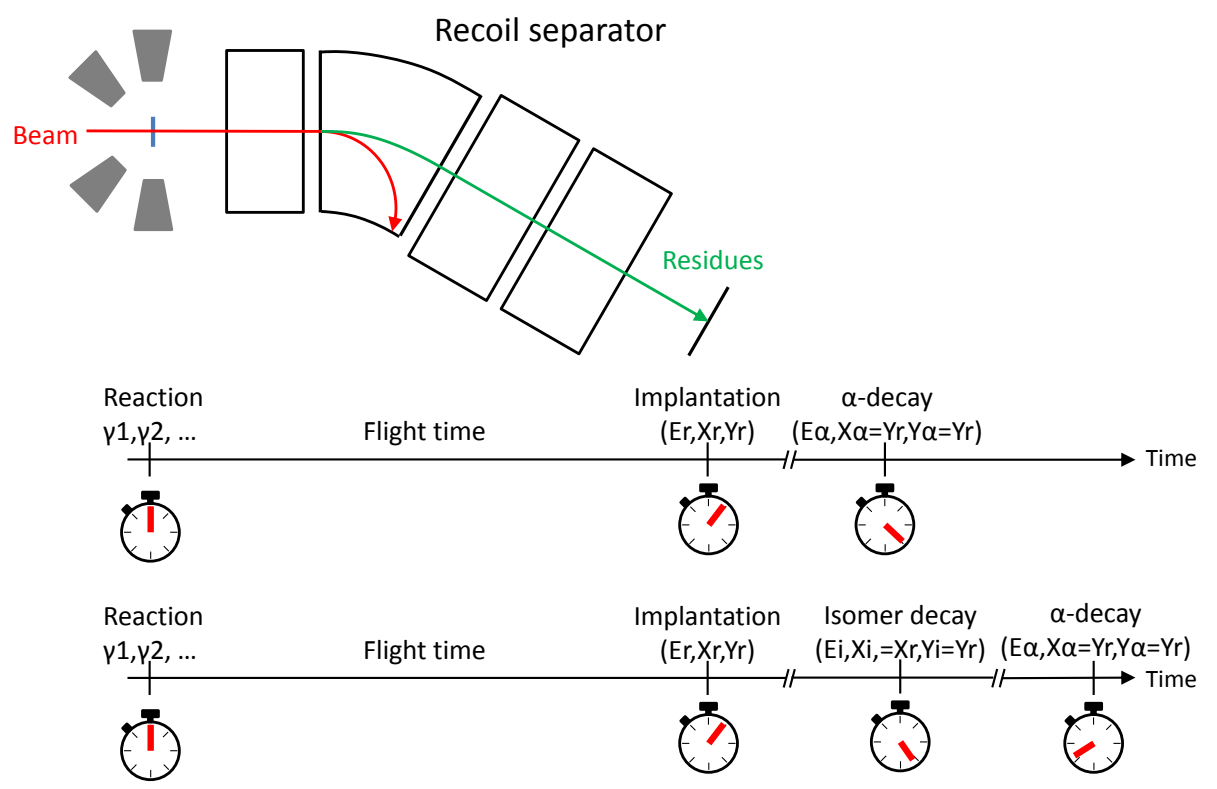

Figure 2: (Color online) Principles of the recoil-decay tagging technique. In the original approach, the nuclei are identified on the basis of kinematics (e.g. time of flight, implantation energy) with additional assignments coming from correlations to the characteristic radioactive (alpha) decay. The correlations allow the prompt radiation emitted at the target at the time of the nuclear reaction to be cleanly extracted from the background of radiation from other processes. In a second variant, the nucleus is implanted in an isomeric state and de-excitation of the isomer follows the implantation and precedes the radioactive (alpha) decay. Selection of this characteristic sequence allows the prompt radiation emitted from the transitions feeding the isomeric state to be isolated. 
the highest $Z$, the reaction target being surrounded by an array of detectors to observe the prompt radiation produced in the nuclear decay process and from interactions of the beam with the target material. When heavy (high$Z$ ) nuclei are studied, most of the cross-section goes into fission and the production of interesting nuclei is extremely rare. In order to select the very few interesting events from the overwhelming background, a recoil separator is employed. The efficiency for detection of fusion-evaporation products can be relatively high, whereas transport of primary beam particles, transfer products and fission fragments is suppressed to a high degree. The focal plane of the recoil separator can be instrumented with a detector system capable of observing a wide range of radiation types (alpha particles, x-rays, gamma rays, conversion electrons) allowing the identification of the fusionevaporation products transported through the separator. The use of delayed coincidences (spatial and temporal correlations) allows the prompt radiation of interest to be extracted from the background of events which are not of interest. More detailed descriptions of the instrumentation available in the leading facilities studying heavy elements can be found in Ref. [1]. The following subsections describe more recent developments and aspects relevant for the current review.

\subsection{Lowering the spectroscopic limit}

In the late 1990s arrays of germanium detectors were teamed with recoil separators such as the Fragment Mass Analyser (FMA) [34] in Argonne National Laboratory (ANL) and the Recoil-Ion Transport Unit (RITU) [35] at the University of Jyväskylä (JYFL) Accelerator Laboratory. These combinations allowed the study of neutron-deficient nuclei in the light $\mathrm{Pb}$ region, and notably of the "transfermium" isotope ${ }^{254}$ No [23, 24]. It was these pioneering studies of ${ }^{254}$ No which really motivated and stimulated the activities reported in this review. Following these first steps in the nobelium region, the majority of in-beam fusion-evaporation experiments have been carried out in JYFL, which is reflected by the content of the current manuscript. Many of these experiments rely on beams of neutron-rich and doubly-magic

${ }^{48} \mathrm{Ca}$ impinging on various heavy targets, such as isotopes of $\mathrm{Hg}, \mathrm{Tl}, \mathrm{Pb}$ and Bi. These reactions have relatively high fusion-evaporation cross-sections, good transmission of products through the separator and the additional advantage that very few (effectively one) reaction channels are populated at a selected beam energy. This allows effective selection of the nuclei of interest with very little background. It is often sufficient to identify the arrival of the 
fusion-evaporation product at the focal plane in order to achieve selection of the prompt radiation of interest. The correlation to subsequent decays is only required to provide further confirmation of the assignment of prompt radiation to a particular nucleus (recoil-gating, as opposed to recoil-decay tagging). Careful analysis and eradication of sources of background, and the introduction of the triggerless "Total Data Readout" data acquisition system [36] in JYFL allowed preliminary studies of nuclei such as ${ }^{255} \operatorname{Lr}$ to be carried out. The cross-section to produce such nuclei is of the order of $200 \mathrm{nb}$, already an impressively low level. These studies required the allocation of up to two weeks of beam time, which is clearly at a premium in most facilities.

The limit in number of nuclei produced resulted from the fact that the maximum counting rate of the germanium detectors had to be kept to a reasonable level in order to maintain spectroscopic quality (resolution, pileup rates, etc). With the traditional "analogue" amplifier-ADC (Analogto-Digital Converter) electronics chain, the counting rates are usually kept at a level of around $10 \mathrm{kHz}$ per detector. This in turn places a limit on the maximum beam intensity that can be used in the experiment. Therefore, the maximum counting rate was typically met with beam intensities of around 10-20 particle nA. In order to overcome these limitations, it has been necessary to improve the electronics used to read out the signals from the germanium detectors. An approach which has now become relatively common is to employ what are colloquially known as "digital" electronics, whereby the signals from the preamplifiers are directly digitised in a flash ADC and further signal processing is performed with a software algorithm in an FPGA (Field Programmable Gate Array). Such an approach generally allows the use of higher counting rates without significant degradation of the resolution, and with less pile-up of signals when compared to "analogue" systems. In JYFL, the JUROGAM array of 43 Compton-suppressed Phase I germanium detectors [37] was initially instrumented with TNT2 14 bit ADC cards with a sampling rate of $100 \mathrm{MHz}$ [38] in 2007. The implementation of digital electronics allowed the maximum counting rates to be increased to the level of $20-30 \mathrm{kHz}$, resulting in successful studies of ${ }^{255} \mathrm{Lr}$ [30] and of high- $K$ states in ${ }^{252}$ No [19]. The average beam currents that could be used were 24 particle $n \mathrm{~A}$ and 30 particle $\mathrm{nA}$, respectively. The results of both of these studies are reported in more detail below.

After these first experiments demonstrated that higher beam intensities could be successfully used and that the yield could be increased, it became clear that studies of nuclei with much lower production cross-sections could 
be attempted. This is particularly true in the case of even-even nuclei, where the gamma-ray intensity is focussed into a single rotational band. A long term goal of this program of studies was to push to higher $Z$ and investigate the development of deformation and collectivity as a function of proton number. A case of particular interest was ${ }^{256} \mathrm{Rf}$, which can be produced with a cross section of approximately $17 \mathrm{nb}$ via the ${ }^{208} \mathrm{~Pb}\left({ }^{50} \mathrm{Ti}, 2 n\right){ }^{256} \mathrm{Rf}$ reaction [31. An additional motivation to study ${ }^{256} \mathrm{Rf}$ is that it is one of the $N=152$ isotones, thus giving an opportunity to study the behaviour of the deformed shell gap at that neutron number. An equally challenging experiment was to study the rotational structure of the neutron-deficient isotope ${ }^{246} \mathrm{Fm}$ [12]. Studies of ${ }^{248,250} \mathrm{Fm}$ had been previously carried out in JYFL, using beams of ${ }^{48} \mathrm{Ca}$ on targets of ${ }^{202} \mathrm{HgS}$ and ${ }^{204} \mathrm{HgS}$, respectively [13, 14, 15. The isotope ${ }^{246} \mathrm{Fm}$ can be produced using the ${ }^{208} \mathrm{~Pb}\left({ }^{40} \mathrm{Ar}, 2 n\right){ }^{246} \mathrm{Fm}$ reaction with a cross-section of $\sim 10 \mathrm{nb}$. Again exploiting the TNT2 digital electronics to instrument what was now the JUROGAM II array of 24 Compton-suppressed clover and 15 Phase I germanium detectors [39], it was possible to once more increase the maximum counting rate in the germanium detectors and hence the maximum beam intensity. A previously unprecedented maximum beam intensity of $\sim 70$ particle $\mathrm{nA}$ was reached, with counting rates of the order of $40 \mathrm{kHz}$ per germanium crystal. On the average, a beam intensity of 40 particle nA was used over the duration of the experiment [12]. Development of a ${ }^{50} \mathrm{Ti}$ beam in 2011 [40] meant that an attempt could be made to study ${ }^{256} \mathrm{Rf}$. At the same time, a new system of digital electronics based on $100 \mathrm{MHz}$ 14-bit ADC cards [41], was installed to instrument JUROGAM II. The system was developed by Daresbury Laboratory and the University of Liverpool as part of the SAGE project, described in more detail in the following section. In September 2011 it was possible to dedicate a total of three weeks of beam time (450 hours) to the study of ${ }^{256} \mathrm{Rf}$, resulting in the observation of the ground-state rotational band in a superheavy nucleus for the first time [31]. Here the term "superheavy" refers to those nuclei whose existence is entirely due to shell effects, a region widely accepted to begin at $Z=104$.

The results of the ${ }^{246} \mathrm{Fm}$ and ${ }^{256} \mathrm{Rf}$ experiments are discussed in more detail in section 3, and prospects for further lowering of the spectroscopic limits are discussed in section 7 .

\subsection{Combined conversion electron and gamma-ray spectroscopy}

When studying deformed nuclei with high values of $Z$, one encounters low-energy $E 2$ transitions which can have a very large internal conversion 
coefficient. In in-beam gamma-ray spectroscopic studies of heavy even-even nuclei, a common feature of the rotational band spectra is that the intensities of the lowest energy radiative transitions in the band do not follow the characteristic shape of the germanium detector efficiency curve. Rather, the intensities of the gamma-ray transitions reduce as the transition energy is reduced and typically the $4^{+}$to $2^{+}$and $2^{+}$to $0^{+}$transitions are not observed. In the rotational band of ${ }^{254} \mathrm{No}$, for example, the $4^{+}$to $2^{+}$and $2^{+}$to $0^{+}$transitions have internal conversion coefficients of 28.8 and 1545, respectively. In odd-mass nuclei, the problem is further exacerbated for the low-energy intra-band $M 1$ transitions which can occur between the strongly-coupled signature partner bands in a well-deformed rotational nucleus. Transitions with $M 1$ character and energies of 100 and $200 \mathrm{keV}$ have internal conversion coefficients of 14.5 and 8.45, respectively, for a proton number of 103 (Lr).

Whilst it has been possible to make measurements of the internal conversion electrons emitted from the decay of heavy nuclei (for example using the SACRED spectrometer at RITU [42]), it is clearly desirable to make a simultaneous measurement of both conversion electrons and gamma rays so that the information available to the experimenter is maximised. Building on the experience gained with the SACRED spectrometer, the concept which became known as the SAGE (Silicon And GErmanium) spectrometer was developed in the mid-to-late 2000s. The SAGE spectrometer was designed and constructed in a collaboration between the University of Liverpool, Daresbury Laboratory and JYFL. The SAGE spectrometer exploits the majority of the germanium detectors from the JUROGAM II array for gamma-ray detection and incorporates a solenoidal magnetic field to transport internal conversion electrons to a segmented silicon detector with 90 individual elements. A schematic drawing of SAGE can be seen in Fig. 3 and a detailed description of the device and performances can be found in Ref. [43].

To date, the SAGE spectrometer has been used in three campaigns of experiments in 2010-2013. In the region of heavy elements, the nuclei ${ }^{249,251} \mathrm{Md}$, ${ }^{253,254}$ No and ${ }^{255} \mathrm{Lr}$ have been studied. The data from these experiments are still under analysis, and results will be published in due course. The potential for combined in-beam gamma-ray and conversion electron spectroscopy is demonstrated by preliminary results obtained in ${ }^{251} \mathrm{Md}$ which are presented in paragraph 4.2. Along with studies of heavy elements, SAGE is also a powerful spectrometer for studies of shape coexistence in the mass 170-190 region and for studies of octupole-deformed nuclei in the mass 220 region. Almost ten experiments have been carried out for studies of this type. 


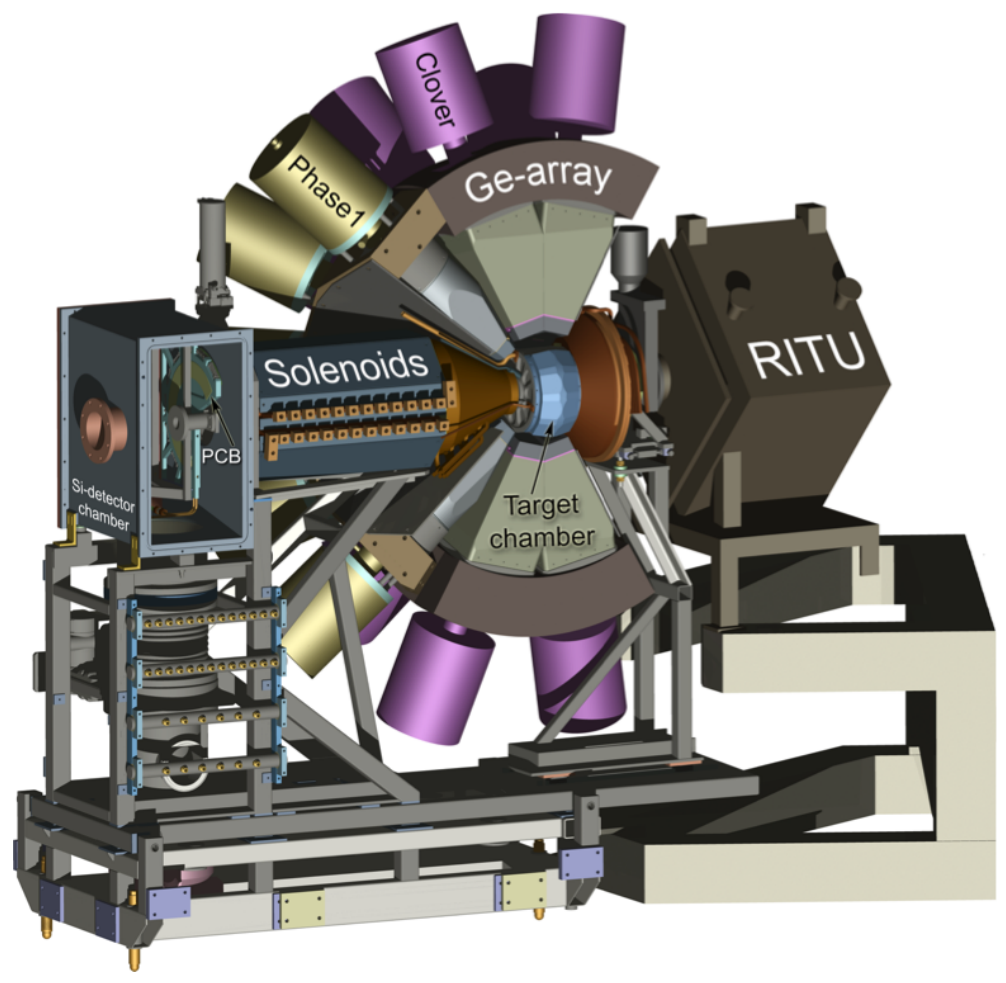

Figure 3: (Color online) Schematic drawing of the SAGE (Silicon And GErmanium) spectrometer installed at the target position of the RITU gas-filled recoil separator in JYFL, Finland. The beam enters from the left and impinges on the target which is surrounded by Compton-suppressed germanium detectors. The internal conversion electrons are transported to a segmented Si detector upstream using a solenoidal magnetic field. 


\subsection{Exploiting transfer reactions}

Historically, transfer reactions such as $(d, p),(d, t)$ and $\left(d, d^{\prime}\right)$ have played a significant role in determining and understanding the structure and singleparticle states of heavy nuclei. A number of studies were carried out in the 1970s using many of the long-lived radioactive targets from $\mathrm{U}$ to $\mathrm{Cf}$ (see, for example, Refs. [44, 45, 46]). The use of transfer reactions is a powerful tool in the characterisation of the populated states, usually allowing clear assignments of the spins and parities, or Nilsson labels in deformed nuclei. The work of Ahmad et al. provides a very nice example of exploiting a neutron stripping $(d, p)$ reaction to populate single-neutron states above the $N=152$ shell gap [45]. Experimental determination of the location of singleparticle states is of importance to benchmark nuclear models. As discussed by many authors, the shell corrections at the next neutron spherical shell gap $N=184$ are sensitive to the locations of the neutron $\mathrm{h}_{11 / 2}, \mathrm{k}_{17 / 2}$ and $\mathrm{j}_{13 / 2}$ states: see [47, 48] and references therein. For protons, the relevant orbitals

are $i_{13 / 2}, i_{11 / 2}$ and $j_{15 / 2}$. In deformed nuclei the low- $K$ components of these states are strongly down-sloping, and can potentially be probed by transfer reactions.

The power and widespread use of transfer reactions is also reflected in Fig. 1, as many of the nuclei where a large number of levels are known are close to $\mathrm{Cm}$ and $\mathrm{Cf}$ isotopes with long half-lives, which allow the production of a target for such studies. Most of the studies from the 1970s to 1990s employed a spectrograph to detect the outgoing particles from the reaction. In recent years, transfer reactions have been used as a tool to populate excited states, the gamma-ray decay of which is then observed in-beam using arrays of germanium detectors. Two main approaches have been used: the first is to use the most neutron-rich and long-lived isotope of a particular element to produce a target, and then to exploit one or two-neutron transfer reactions with light ions to populate excited states in more neutron-rich isotopes. Such experiments can be carried out to good effect even using a rather modest setup of a few germanium detectors surrounding the target. It is also possible to use silicon detectors to detect the outgoing beam-like particles in coincidence with the germanium detectors, thus allowing the identification of the first few excitations ( up to $\approx 12 \hbar$ ) to be mapped in the most neutron-rich isotopes [49]: see Fig. 4 as an example. The second approach involves using deep-inelastic and/or transfer reactions, in conjunction with Coulomb excitation, with very heavy beams to populate the nuclei of interest. Often a thick target is used with all reaction products stopped in the target, and the gamma rays detected 

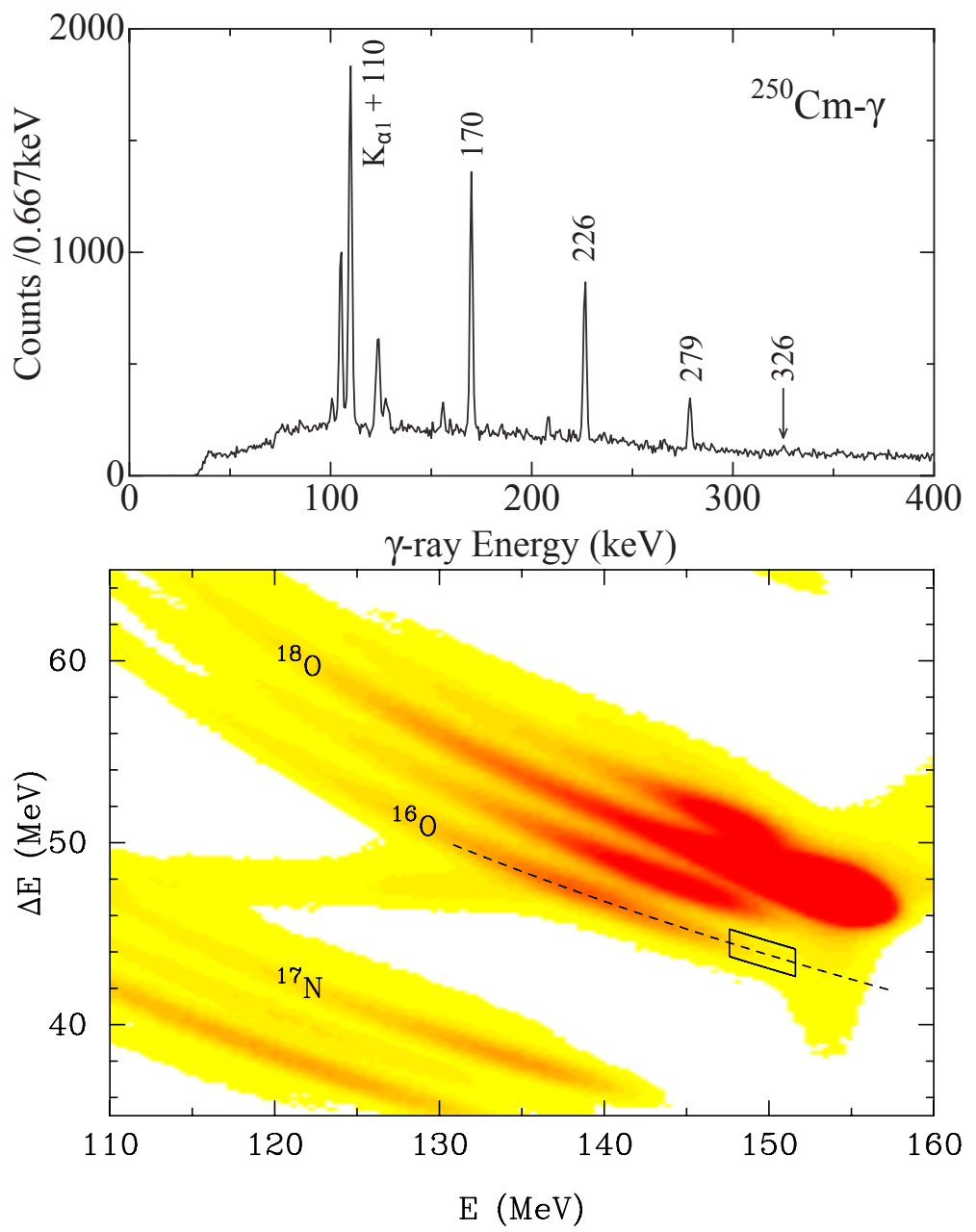

Figure 4: (Color online) Lower panel: $E-\Delta E$ plot of scattered particles in the reaction ${ }^{248} \mathrm{Cm}\left({ }^{18} \mathrm{O},{ }^{16} \mathrm{O}\right){ }^{250} \mathrm{Cm}$ at $162 \mathrm{MeV}$ bombarding energy. The enclosed area with solid lines corresponds to an excitation energy in ${ }^{250} \mathrm{Cm}$ between 0 and $4 \mathrm{MeV}$. Upper panel: Gammaray spectrum of ${ }^{250} \mathrm{Cm}$, obtained by selecting those particles indicated by the enclosed area in the lower panel. Figure reprinted from [49] with permission of the Physical Society of Japan. 


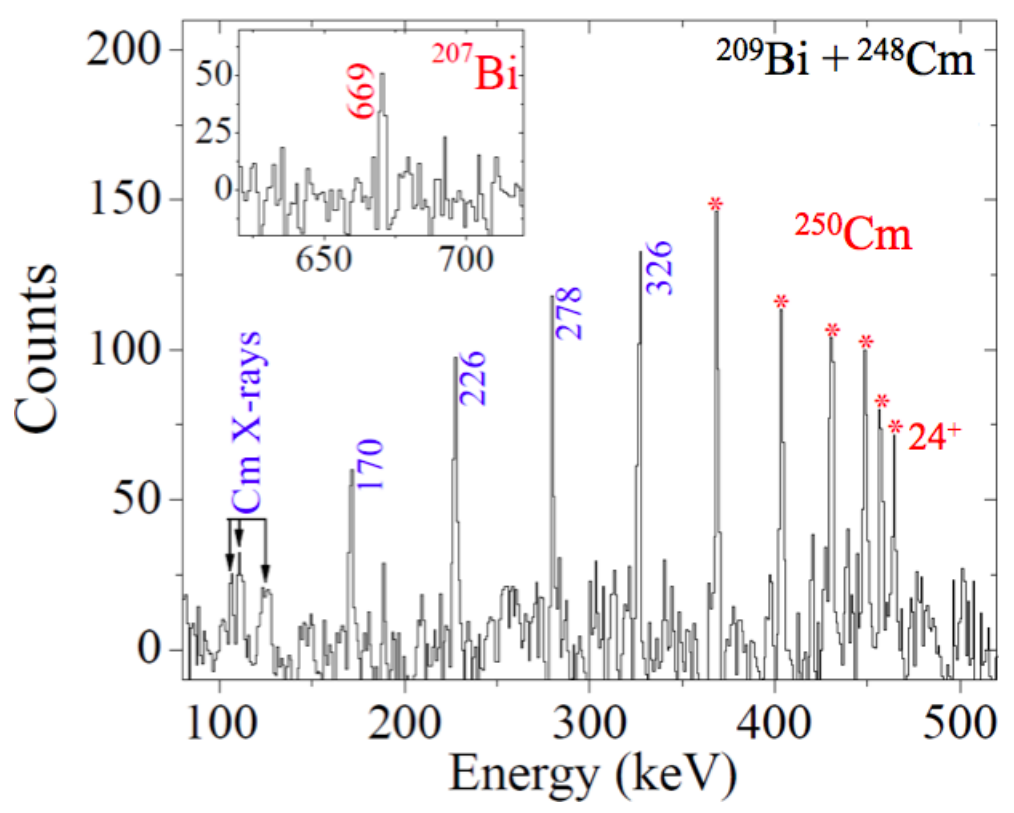

Figure 5: (Color online) The spectrum of two-neutron transfer to high spins to access the highest neutron orbitals in the $N=154$ nucleus ${ }^{250} \mathrm{Cm}$, obtained from a sum of triple-gates in a GAMMASPHERE hypercube. The nucleus is populated to at least twice the angular momentum as compared to an ${ }^{18} \mathrm{O}$ beam. Coincidences with $\mathrm{Cm}$ x-rays are prominent. Inset shows cross-coincidence with two-neutron transfer from the ${ }^{209} \mathrm{Bi}$ beam. Taken from Hota, Ph.D. thesis [9]. 
with powerful multi-detector arrays like GAMMASPHERE. These experiments can build on the first few states identified in the first approach and extend the knowledge of excited states in these nuclei to significantly higher angular momenta around $\approx 24 \hbar$ [4, 9]. The reaction mechanism can populate a large number of nuclei, resulting in extremely complicated gamma-ray spectra. In addition, the most neutron-rich nuclei in this region are radioactive with long half-lives, and pose significant experimental challenges in terms of signal-tonoise. Thus, in order to extract the information on a particular nucleus, the power of a large array of germanium detectors is essential for effective use of multi- and cross-coincidence techniques. In both approaches, the nuclei under study are often impossible to study in-beam by any other means. The most neutron-rich isotopes of Th $(A=236), \mathrm{U}(A=242), \mathrm{Pu}(A=246), \mathrm{Cm}$ $(A=250)$ and $\mathrm{Cf}(A=252)$ for which there is excited state data available were all studied by means of two-neutron transfer reactions (see, for example, [9, 49]). Thus, while the highest-lying proton orbitals must be probed using fusion-evaporation reactions, access to the highest-lying neutron orbitals are via neutron transfer reactions using radioactive neutron-rich targets.

Inelastic cross-sections in this region, which can vary from a few tens to over $100 \mathrm{mb}$, are significantly higher than the fusion-evaporation crosssections mentioned for $Z>100$ nuclei, with each neutron transfer lowering the cross-section by at least an order of magnitude. Thus, while these experiments are simpler in terms of the experimental set-up, the extraction of the signal from the noise requires a combination of techniques which include (a) cross-coincidence with the binary reaction partner to identify the nuclide, (b) $\mathrm{x}$-gamma coincidences for $Z$-identification, (c) gating on sum-energy and fold to reduce fission background, and (d) double- or triple-gamma coincidence techniques to isolate the near-identical rotational bands in this deformed region (see Fig. 5). These techniques have allowed high-spin studies of the $N=152$ nuclei ${ }^{246} \mathrm{Pu}$ and ${ }^{250} \mathrm{Cf}$, the $N=153$ nucleus ${ }^{251} \mathrm{Cf}$ [11], and the $N=154$ nucleus ${ }^{250} \mathrm{Cm}$ [9, 49].

\subsection{Fission barriers from entry distributions}

When discussing the stability of heavy nuclei, one of the most important parameters is clearly the fission barrier which is created due to the shell effects. The fission barrier height is also an important factor in the production cross section when a heavy nucleus is produced in a nuclear reaction, as it determines the survival against fission. Very often, both decay and in-beam spectroscopic studies are more focussed on determining the nuclear structure 
properties (such as single-particle energies, etc) which underlie and generate the shell effects. Relatively few studies are focussed on a direct measurement of the fission barrier height. Such measurements can be extremely valuable in constraining nuclear theory, for which there are considerable differences in the predictions of barrier heights because of discrepancies in the underlying single-particle spectrum and, hence, the shell-corrected binding energy. Therefore, a direct measurement of the barrier height provides an incisive test of theory - the predicted fission barrier heights in ${ }^{254}$ No vary from around 6 - $13 \mathrm{MeV}$, depending on the approach used.

A measurement of the fission barrier height can be most directly obtained by determining the fission probability as a function of excitation energy in transfer reactions, i.e. from the fission threshold [50]. The use of transfer reactions clearly requires production of a suitable long-lived target, making this approach close to impossible beyond $Z=98$. A complementary method to determine the fission probability exploits the fact that, below the fission threshold, $P_{\text {fission }}=1-P_{\gamma}$. Thus a measurement of the gamma-ray emission probability $P_{\gamma}$ gives a measurement of $P_{\text {fission }}$, and the precipitous drop of $P_{\gamma}$ (from 1) near and above the saddle energy serves as a proxy for the fission threshold.

The gamma-ray emission probability $P_{\gamma}$ can be determined from the socalled "entry distribution", which represents the points in spin and excitation energy $\left(I, E^{*}\right)$ from which gamma-ray decay towards the ground state begins. The argument is that if a point can be measured in the $\left(I, E^{*}\right)$ plane, then the nucleus must have survived fission. Near and above the saddle energy, fission will truncate the entry distribution.

In order to determine the entry distribution $\left(I, E^{*}\right)$, the summed energy and multiplicity of gamma-rays emitted in the decay of a nucleus produced via a fusion-evaporation reaction is measured. The measurements require that around the reaction target, as much of $4 \pi$ as possible is covered in order to maximise the efficiency for collection of gamma-rays. A very careful evaluation of the detector response function and an unfolding procedure is required in order to convert the measured sum energy and multiplicity into a plot of spin versus excitation energy. Experiments of this type were carried out for the first time beyond $Z=98$ using the GAMMASPHERE array in the late 1990s [25]. 


\section{Even-Even Nuclei}

As mentioned in the introduction to this review, over the past decade or so a significant number of new in-beam spectroscopic studies have been performed to study nuclei in this region from plutonium to rutherfordium, over a wide range of neutron number. Two main foci can be identified in the goals of these experiments: systematic studies of shell effects and pairing, in particular in relation to the deformed shell gaps at $Z=100$ and $N=152$, and investigation of the high-spin rotational behaviour, in particular the role played by $\mathrm{i}_{13 / 2}$ protons and $\mathrm{j}_{15 / 2}$ neutrons in the alignment properties of rotational bands. Details of the experiments and level assignments in eveneven isotopes of curium, californium, fermium and nobelium can be found in ref. [1. The following discussions and references provided are not meant to be exhaustive, and cover only those works of direct relevance to the discussion, or which have been published after the previous review.

\subsection{Shell effects}

The deformed shell gap at neutron number $N=152$ has been the subject of experimental investigation for several decades, and is now well established. In recent years, it has, however, been possible to extend knowledge of the behaviour of the gap over a much wider range of proton number. In the plutonium isotopes, the $N=152{ }^{246} \mathrm{Pu}$ was first studied via $(t, p)$ reactions on ${ }^{244} \mathrm{Pu}$ [51] and more recently by two-neutron transfer using beams of ${ }^{18} \mathrm{O}$ [52] and ${ }^{208} \mathrm{~Pb}[9]$. Using four sets of silicon $\Delta E-E$ telescopes and an array of six germanium detectors, the ground-state band was established up a spin of $12 \hbar$ with the ${ }^{18} \mathrm{O}$ beam [52]. Building on this work, the level scheme was extended to $\approx 20 \hbar$ with a ${ }^{208} \mathrm{~Pb}$ beam and the power of the GAMMASPHERE array 9 .

In the curium isotopes, ${ }^{248} \mathrm{Cm}$ (along with several other isotopes) has been investigated to high spin via inelastic scattering due the long half-life which enables production of a target. In the californium isotopes, the halflives are much shorter than in the curium isotopes, making production of a target and in-beam studies with germanium detectors much more difficult due to the high count rate induced by the radioactive decay of the target. In spite of these difficulties, it has been possible to make transfer reaction and Coulomb excitation studies with Cf targets. The longest-living isotopes are ${ }^{249,250,251} \mathrm{Cf}$ with half-lives of $351,13.1$ and 898 years, respectively. In the

work of Takahashi et al. [10], an ${ }^{18} \mathrm{O}$ beam was used to bombard a Cf target 
consisting of $63 \%{ }^{249} \mathrm{Cf}, 13 \%{ }^{250} \mathrm{Cf}$ and $24 \%{ }^{251} \mathrm{Cf}$. Using the same set of silicon and germanium detectors as described above (paragraph 2.3), it was possible to identify the ground-state bands of ${ }^{248,250,252} \mathrm{Cf}$ up to spins of 10 , 12 and $10 \hbar$, respectively. Using similar targets, these bands in ${ }^{248,250} \mathrm{Cf}$ have again been extended to angular momenta in the mid-twenties using heavy beams and GAMMASPHERE 9 .

The fermium isotopes are of interest as, with $Z=100$, their proton number coincides with another deformed shell gap. The fermium isotopes have been studied using fusion-evaporation reactions and data on excited states is now available from neutron number $N=146$ to $N=156$. The most neutrondeficient isotope to be studied was the focus of the experiment described earlier, in which the ${ }^{208} \mathrm{~Pb}\left({ }^{40} \mathrm{Ar}, 2 n\right){ }^{246} \mathrm{Fm}$ reaction was used to delineate the ground-state rotational band up to a spin of $16 \hbar$ [12]. By using targets of ${ }^{202,204} \mathrm{HgS}$ with beams of ${ }^{48} \mathrm{Ca}$, the ground-state bands of ${ }^{248,250} \mathrm{Fm}$ have been established to spins of up to 14 and $22 \hbar$, respectively [13, 15]. Unfortunately, it has not been possible to make a high-spin study of the particularly interesting isotope ${ }^{252} \mathrm{Fm}$, which has both proton and neutron number corresponding to a deformed shell gap $(Z=100$ and $N=152)$. However, the energy of the $2^{+}$ state was recently determined from high-resolution alpha decay spectroscopy from the decay of ${ }^{256} \mathrm{No}[53]$.

In the nobelium isotopes, essentially no new studies of the properties of the ground-state bands have been performed since the review of Herzberg and Greenlees [1]. However, there have been experiments focused on characterisation of the known high- $K$ states in ${ }^{252,254}$ No (see section 5).

One of the highlights of the past few years was the study of ${ }^{256} \mathrm{Rf}$ discussed above. Using the ${ }^{208} \mathrm{~Pb}\left({ }^{50} \mathrm{Ti}, 2 n\right){ }^{256} \mathrm{Rf}$ reaction it was possible to delineate the ground-state rotational band up to a spin of $20 \hbar$. The fission-tagged gammaray singles spectrum of ${ }^{256} \mathrm{Rf}$ is shown in Fig. 6. The results of this experiment are discussed in more detail in the next section.

The availability of systematic data can be exploited to draw conclusions about the effects of the deformed shell closures on the structure of the eveneven nuclei in the region of $Z=100$ and $N=152$. One of the simplest ways to reveal these effects is to look at the excitation energies of the first $2^{+}$states. The energies of the $2^{+}$states are sensitive to the deformation and to shell effects, as at a shell gap it is expected that the pairing correlations are reduced, leading to an increase in the moment of inertia (see, for example [54]). The current knowledge of the excitation energies of the first $2^{+}$states from plutonium to rutherfordium is shown in Fig. 7. The data have been taken 


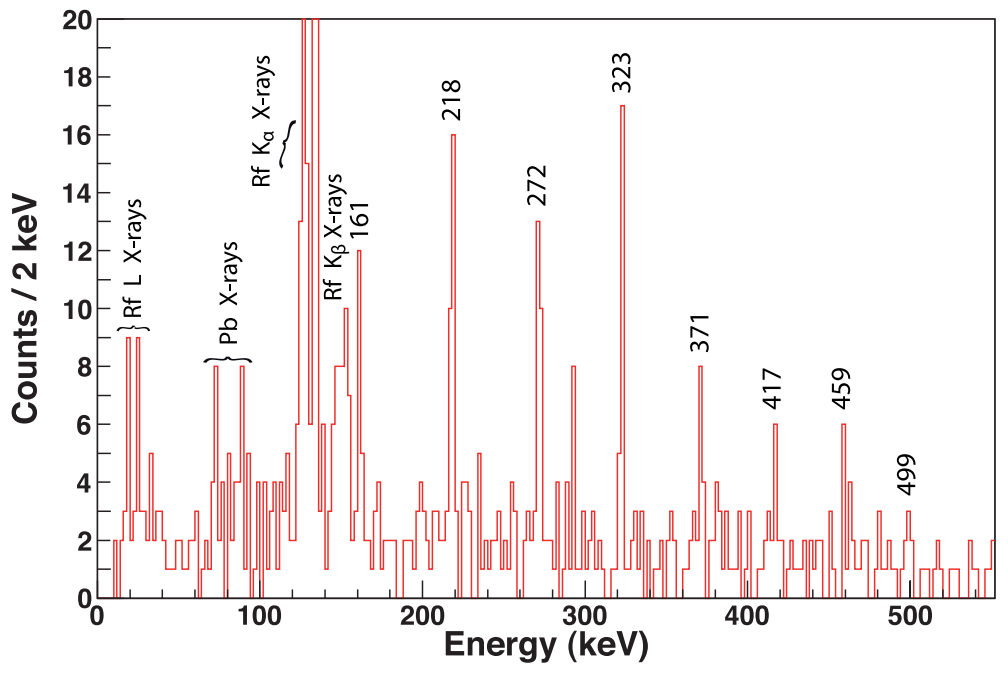

Figure 6: (Color online) Spectrum of singles gamma rays tagged with ${ }^{256} \mathrm{Rf}$ recoils tagged by fission events within a search time of $100 \mathrm{~ms}$. Taken from Greenlees et al. [31, Creative Commons Attribution (http://creativecommons.org/licenses/by/3.0/).

from the ENSDF table [55], or from published data, except for ${ }^{252} \mathrm{Fm}$ which is taken from Ref. [53]. It should be noted that in several cases, the energy of the $2^{+}$state is determined from a "Harris" fit to the higher spin members of the rotational band. This is due to the fact that it is not possible to directly measure the energies due to internal conversion, as described earlier. The experimental kinematic moment of inertia $\mathcal{J}^{(1)}=\hbar^{2}(2 I-1) / E_{\gamma}(I)$ and dynamic moment of inertia $\mathcal{J}^{(2)}=4 \hbar^{2} /\left[E_{\gamma}(I)-E_{\gamma}(I-2)\right]$ can also be parameterised in terms of the kinematic and dynamic moments of inertia according to the formalism of Harris [56], whereby:

$$
\begin{aligned}
& \mathcal{J}^{(1)}=\mathcal{J}_{0}+\mathcal{J}_{1} \omega^{2}, \\
& \mathcal{J}^{(2)}=\mathcal{J}_{0}+3 \mathcal{J}_{1} \omega^{2},
\end{aligned}
$$

where $\omega$ is the rotational frequency $\left(E_{\gamma} / 2\right)$. After fitting the Harris parameters $\mathcal{J}_{0}$ and $\mathcal{J}_{1}$ it is then possible to extrapolate and determine the energies of the unobserved $4^{+}$to $2^{+}$and $2^{+}$to $0^{+}$transitions using the formula (restricted to the case of $K=0$ and with no alignment):

$$
I=\mathcal{J}_{0} \omega+\mathcal{J}_{1} \omega^{3}+1 / 2,
$$

where $I$ is the initial spin for the transition. According to the expectations based on consideration of pairing and the effect on the moment of inertia, it 
should be expected that minima in the excitation energies of the $2^{+}$states should be seen at $N=152$ and $Z=100$. As can be seen from the upper panel of Fig. 7, only in the fermium isotopes is it possible to follow the trend across the full span from $N=146$ to $N=156$. However, even with the more limited data for other elements, some conclusions can be drawn. As discussed in the work of Makii et al., it appears that the shell gap at $N=152$ is weakened [52. This is reflected in the rather high energy of the $2^{+}$state in ${ }^{246} \mathrm{Pu}$. This weakening of the $N=152$ gap is also evidenced by the decreased energy of the $\nu 1 / 2^{+}[620]$ state and the behaviour of the deduced single-particle energies for lower $Z$ in the work of Qian et al. [57]. In the curium and californium isotopes, the minimum seems to be at $N=150$ rather than $N=152$. For the fermium isotopes, there is a clear minimum at $N=152$, corresponding to ${ }^{252} \mathrm{Fm}$ also with $Z=100$. The limited data for nobelium isotopes also has the minimum at $N=152$, though more systematic studies would be desirable. The lower panel of Fig. 7 shows the corresponding data plotted for isotopes as a function of proton number. In this plot the minimum should be expected at $Z=100$, where the deformed shell gap is located. It is interesting to note that for $N=152$ and the available data for $N=154$ the minimum is indeed at $Z=100$. However, for $N=148$ the minimum seems to be shifted to $Z=98$ where no significant gap is expected. Indeed, ${ }^{248} \mathrm{Cf}$ exhibits the lowest $2^{+}$energy of all. To date there is no theoretical explanation for these observations.

As discussed in detail elsewhere in this volume, additional insight into the shell effects can come from inspection of the atomic masses, or as shown in Figs. 8 and 9 from the neutron and proton separation energies and the related shell gap parameter. It is of interest to compare these figures to the excitation energies of the $2^{+}$states. As emphasised in the lower panel of Fig. 8, the deformed shell gap at $N=152$ is clearly seen for all isotopes from curium to nobelium. However, when the proton separation energies are inspected, it can be seen that for $N=148$ and $N=150$ the shell effect is strongest at $Z=98$. This correlation between the $2^{+}$energies and the masses and the effect at $Z=98$ is not yet understood.

\subsection{Moments of inertia and rotational alignments}

The moments of inertia and rotational alignment are sensitive to nuclear properties such as the pairing strength and to the specific orbitals active at the Fermi surface, thus systematic analysis of the moments of inertia can provide invaluable information on such properties. The recent experiment to study ${ }^{256} \mathrm{Rf}[31$ ] provided new data which could be compared to that of 

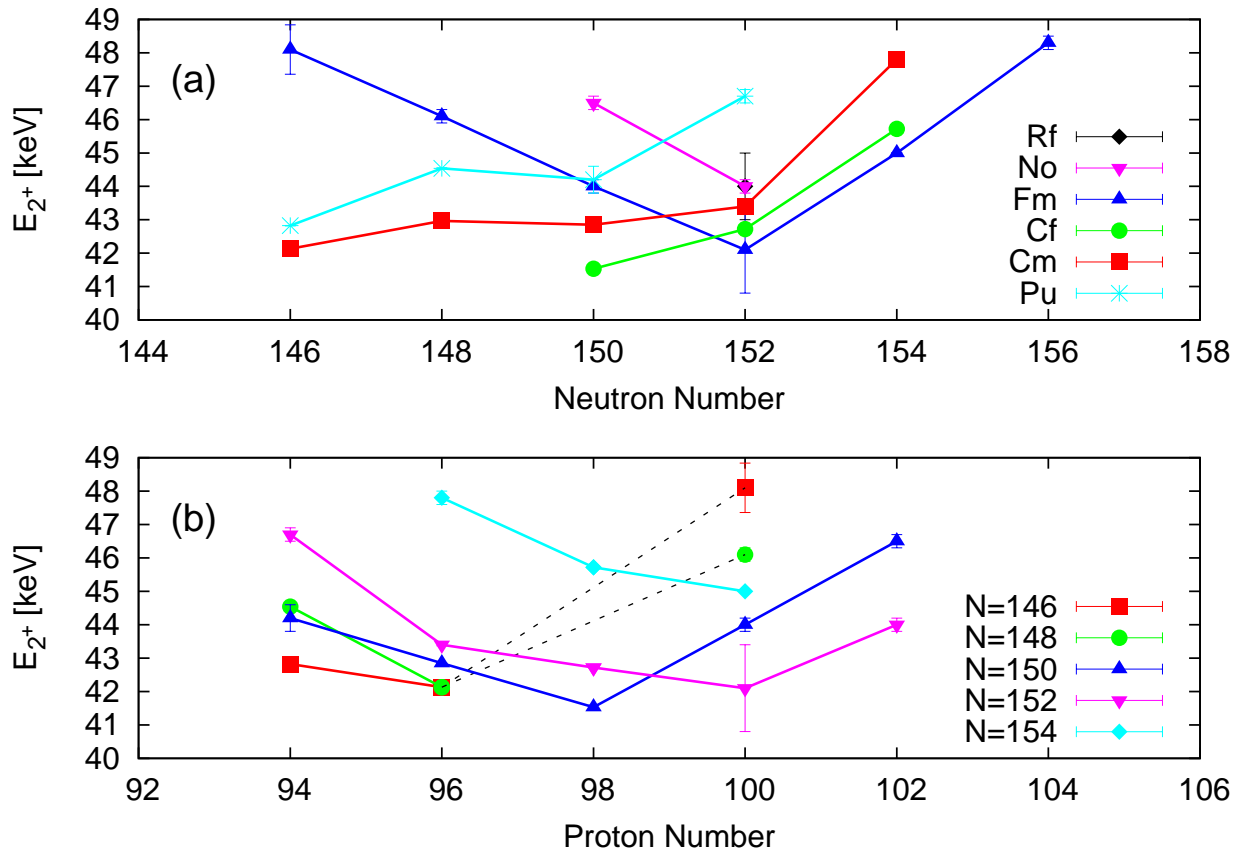

Figure 7: (Color online) Systematic behaviour of the energies of the first $2^{+}$states in even-even nuclei. Upper panel: For isotopes of plutonium to rutherfordium with neutron number from 146 to 156 . Lower panel: For isotopes with neutron numbers from 146 to 154 from plutonium to nobelium. Data are taken from the ENSDF table [55], or from published data, except for ${ }^{252} \mathrm{Fm}$ which is taken from Ref. 53. 


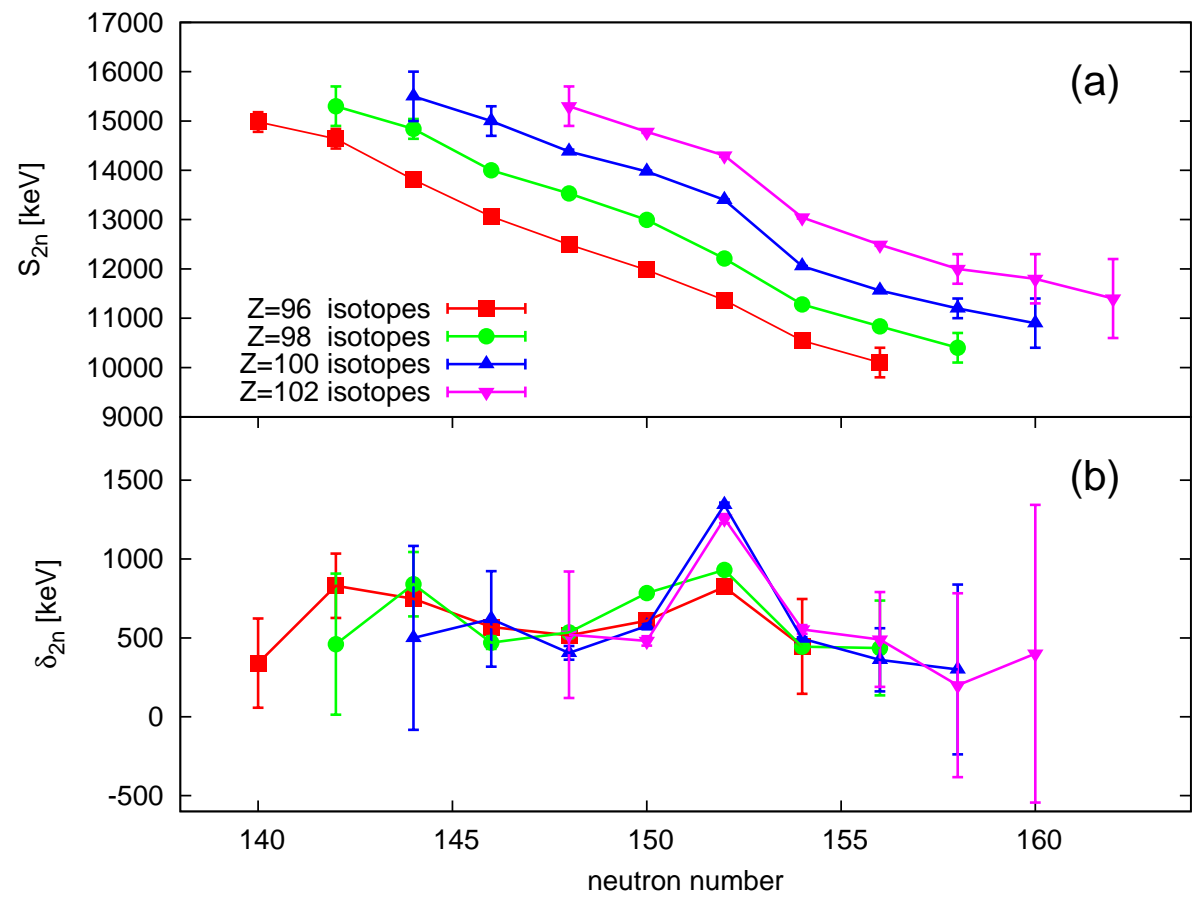

Figure 8: (Color online) Upper panel: Systematic behaviour of the two-neutron separation energies for isotopes of curium, californium, fermium and nobelium. Lower panel: Shell gap parameter $\delta_{2 n}$ for the same sequence of isotopes. Data are taken from the 2003 Atomic Mass Evaluation [58, and the figure from the $\mathrm{PhD}$ thesis of Ketelhut [13. 


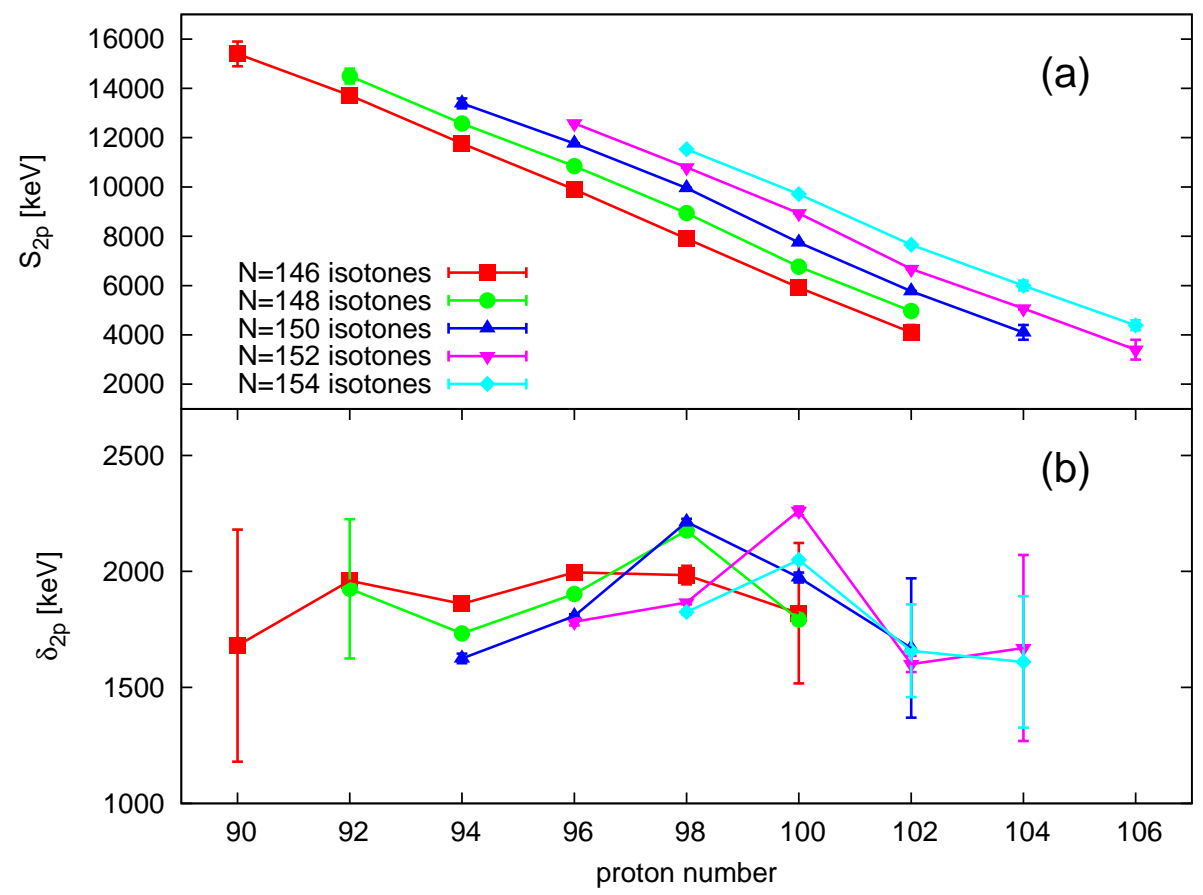

Figure 9: (Color online) Upper panel: Systematic behaviour of the two-proton separation energies for isotones of with neutron number from 146 to 154 . Lower panel: Shell gap parameter $\delta_{2 p}$ for the same sequence of isotones. Data are taken from the 2003 Atomic Mass Evaluation [58, and the figure from the $\mathrm{PhD}$ thesis of Ketelhut [13]. 


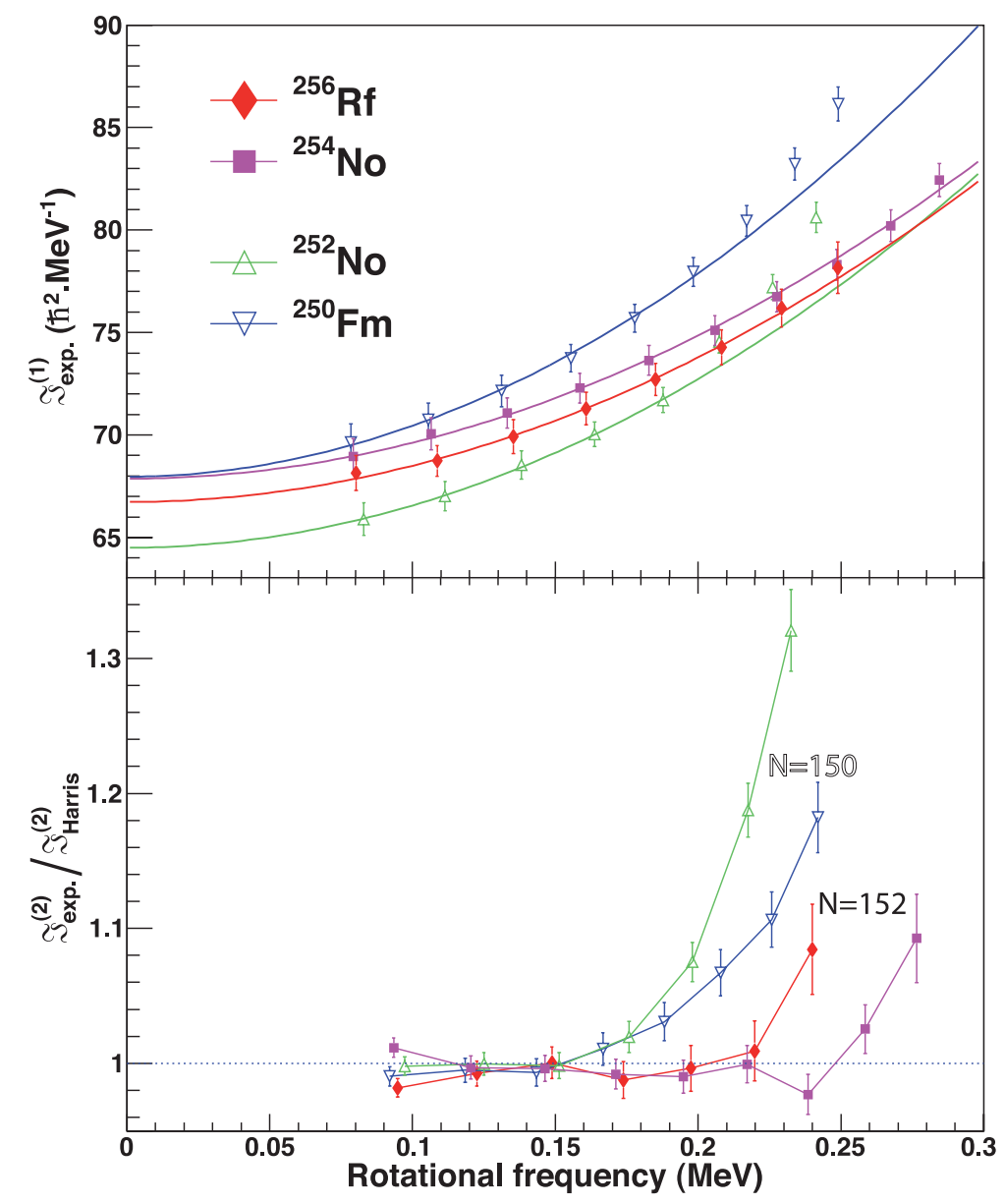

Figure 10: (Color online) Upper panel: Kinematic moment of inertia as a function of rotational frequency for $N=150$ and $N=152$ isotones. The lines are drawn according to fits of the Harris parameters. Lower panel: Dynamic moment of inertia of the $N=150$ and $N=152$ isotones, normalized to that calculated using the fitted Harris parameters. Taken from Greenlees et al. [31, Creative Commons Attribution (http://creativecommons. org/licenses/by/3.0/). 
lighter nuclei. The kinematic moment of inertia as a function of rotational frequency for ${ }^{256} \mathrm{Rf}$ is shown in the upper panel of Fig. 10 and compared to that of ${ }^{250} \mathrm{Fm},{ }^{252} \mathrm{No}$ and ${ }^{254} \mathrm{No}$. The lines drawn are from Harris fits to the low-spin part of the data and plotted according to equation 1. The moment of inertia of ${ }^{256} \mathrm{Rf}$ shows very similar behaviour as a function of rotational frequency as the $N=152$ isotone ${ }^{254}$ No, though the absolute value is slightly lower over the full frequency range.

As has been noted previously, the rotational properties of the $N=150$ isotones are somewhat different, showing much faster alignment than the $N=152$ nuclei. It is interesting to question the differences in absolute value of the moments of inertia in these nuclei. It can be seen that whilst the $N=150$ isotones show similar alignment behaviour, ${ }^{250} \mathrm{Fm}$ has a larger moment of inertia than ${ }^{252}$ No. At low frequency, ${ }^{254}$ No has a similar moment of inertia to ${ }^{250} \mathrm{Fm}$, but slightly higher than that of ${ }^{256} \mathrm{Rf}$. The differences can again be related to the deformed shell gaps and the effect on pairing and the moment of inertia. The nucleus ${ }^{252}$ No has the lowest moment of inertia, which having 102 protons and 150 neutrons has the Fermi surface just above the $Z=100$ and just below the $N=152$ deformed shell gaps. When two protons are removed to get ${ }^{250} \mathrm{Fm}$, the moment of inertia increases, which may be a reflection of the reduced pairing correlations due to the $Z=100$ shell gap. A similar argument goes for ${ }^{254} \mathrm{No}$, in which the moment of inertia is larger when two neutrons are added compared to ${ }^{252}$ No. Again, this is evidence of the influence of the $N=152$ shell gap. Following these arguments, one would expect that ${ }^{252} \mathrm{Fm}$ with $Z=100$ and $N=152$ should have the highest moment of inertia and lowest $2^{+}$energy, as seen in Fig. 7. It might also be expected that if there is a significant shell gap at $Z=104$, the moment of inertia of ${ }^{256} \mathrm{Rf}$ would be larger than that of the isotone ${ }^{254}$ No. As can be seen from Fig. 10 . this is not the case. In fact the moment of inertia is slightly lower, which may be indicative of the diminishing influence of the $Z=100$ shell gap. It is also suggestive that there is no significant deformed shell gap at $Z=104$ [31]. The lower panel of Fig. 10 shows the experimental dynamic moment of inertia as a function of rotational frequency for the $N=150$ and 152 isotones, normalized to the smoothly-behaving $\mathcal{J}^{(2)}$ from the Harris fit. The behaviour at low rotational frequency is similar for all nuclei, but a divergence from the smooth behaviour appears above frequencies of around $\hbar \omega=0.15 \mathrm{MeV}$ in the $N=150$ isotones and above $0.2 \mathrm{MeV}$ in the $N=152$ nuclei, indicating an alignment effect. It is interesting to note that the alignment occurs simultaneously in the $N=150$ isotones, but appears later in the $N=152$ isotones and is delayed 
in ${ }^{254}$ No relative to ${ }^{256} \mathrm{Rf}$. It is well-known that in this region of nuclei there is competition between neutron $j_{15 / 2}$ and proton $i_{13 / 2}$ alignment effects. These effects are sensitive to the relative positions of the relevant orbitals to the Fermi surface.

As new experimental data on rotational bands has been collected, in parallel a large number of theoretical works using different approaches have tried to reproduce the systematic behaviour observed. Common themes can be found in many of these theoretical works - reproduction of the systematic behaviour of the moments of inertia and investigation of the pairing strengths and associated three point-mass differences. Discussion of the general findings of these works can be found in section 6. The competition between neutron $\mathrm{j}_{15 / 2}$ and proton $\mathrm{i}_{13 / 2}$ alignment effects is a long-standing question which has only partially been answered in the actinide and transfermium region. This is partly due to the fact that in many cases, the rotational bands are only known to medium spins of (at best) 10-20 . The associated rotational frequencies at these spin values are below those where alignment effects are predicted. Clarification of which orbitals are responsible for the alignment effects is also complicated by the fact that the alignments may occur simultaneously, or that the alignment may be delayed or smoothed by effects such as octupole correlations. A clear case of this is observed in the $\mathrm{Pu}$ isotopes, where ${ }^{242,244} \mathrm{Pu}$ show a well-defined upbend due to the alignment of $i_{13 / 2}$ protons, but in ${ }^{240} \mathrm{Pu}$ the upend is not seen due to octupole correlations [59]. This will be discussed further in section 6.1.

\subsection{Fission barrier of ${ }^{254} \mathrm{No}$}

As previously mentioned, the entry distribution of ${ }^{254}$ No was first measured following the pioneering study of the ground-state rotational band using GAMMASPHERE coupled to the FMA in the late 1990s [23, 25]. The reaction ${ }^{208} \mathrm{~Pb}\left({ }^{48} \mathrm{Ca}, 2 n\right){ }^{254}$ No was employed with beam energies of 215 and $219 \mathrm{MeV}$. Using higher beam energies allows the entry distribution to be mapped to higher spin and excitation energy, but in the case of ${ }^{254} \mathrm{No}$ a compromise must be made between increased excitation energy and reduced yield. This is due to the fact that the excitation function is rather narrow, and the yield drops quickly with increasing beam energy. The study of Reiter et al. 25] concluded that the fission barrier must have a height of at least $5 \mathrm{MeV}$ and that the shell-correction energy persists to high spin. More recently, the experiment was repeated using higher beam energies of 219 and $223 \mathrm{MeV}$ in order to populate to even higher excitation energy and spins [29]. At 

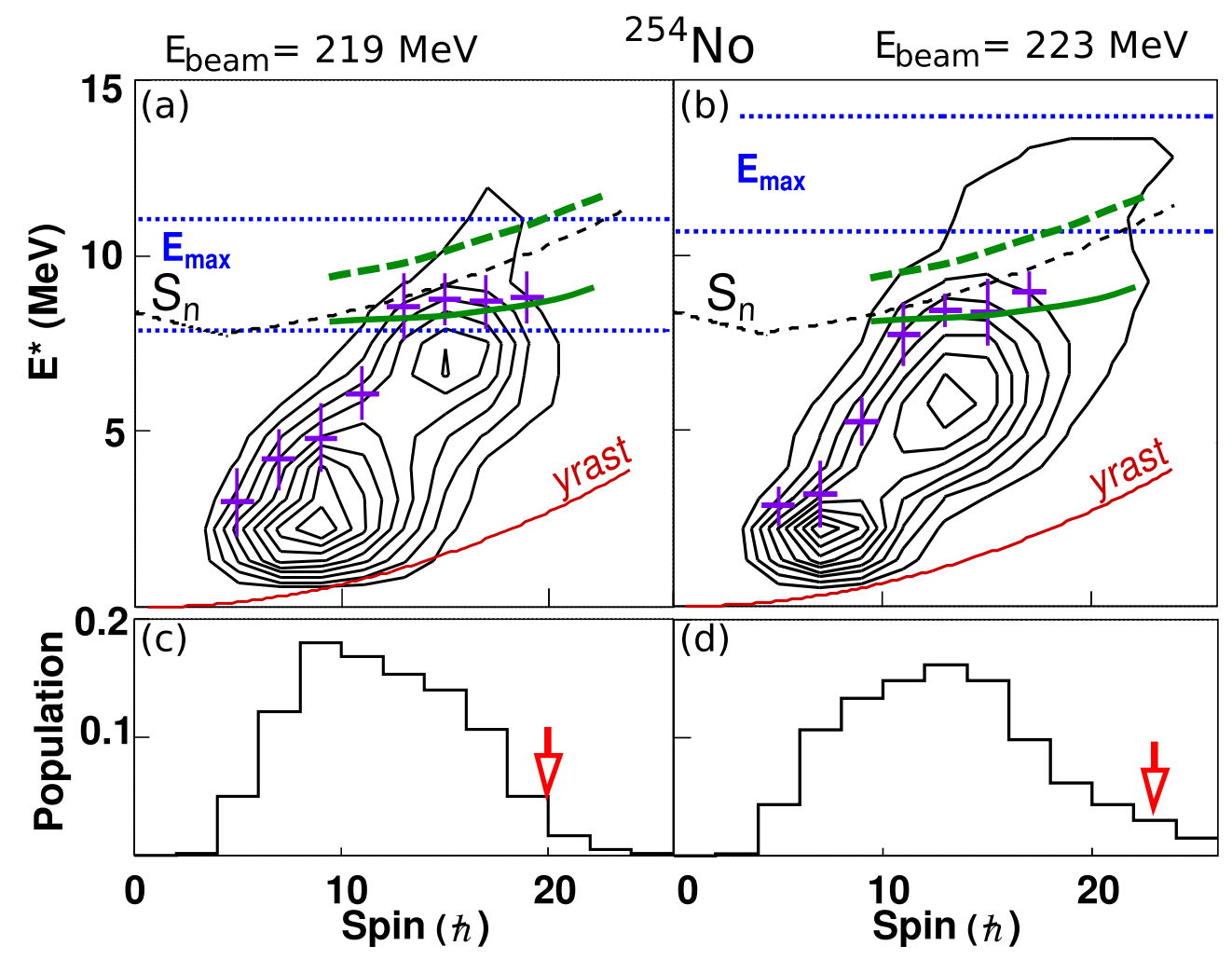

Figure 11: (Color online) Entry distributions for $E_{\text {beam }}=219$ (a) and $223 \mathrm{MeV}$ (b) obtained in the work of Henning et al. 29]. Each contour line corresponds to a decrement of $10 \%$ of the maximum population. The yrast line and neutron separation energy are represented by the solid red and dotted black lines, respectively. The maximum possible excitation-energy range is represented by the blue dotted lines, with the higher (lower) value corresponding to a reaction at the front (back) of the target. The half-maximum point for each spin slice is marked in purple with error bars. The solid and dashed green lines between 10 and $22 \hbar$ represent the $E_{1 / 2}$ values predicted with the deduced value for $B_{f}$ and with $B_{f} 2 \mathrm{MeV}$ higher. Panels (c) and (d) show the corresponding spin projections of the distributions. The points where the spin distributions fall to $10 \%$ of their maximum are marked with a red arrow. Reprinted with permission from Henning et al., Phys. Rev. Lett. 113, 262505 (2014) by the American Physical Society. 
$219 \mathrm{MeV}$, there is a Q-value constraint on the maximum allowable energy, which means that the full effect of the fission barrier cannot be mapped. At $223 \mathrm{MeV}$ there is no such constraint, yet there was no increase in the energy of the entry distribution: a clear saturation effect on the population of the entry states was visible. The entry distribution was cut by the onset of fission near the top of the fission barrier. With further analysis of the data and some aid from statistical decay calculations, the height of the fission barrier was determined: 6.6(5) $\mathrm{MeV}$ at spin 0 and $6.0(9) \mathrm{MeV}$ at spin 15 [29]. The experimental value for the fission barrier was used to confront the predictions from theory. In the case of density functional theory, the predicted barriers (with Skyrme and Gogny D1S interactions) are of the order of 9-13 MeV, whilst that from macroscopic-microscopic predictions are $6.8 \mathrm{MeV}$, in better agreement with the experiment (see ref. [29] and references therein). These discrepancies in the barrier height predictions can be partially ascribed to the differences in the underlying single-particle energies, a recurring theme which is discussed in more detail in section 6 .

\section{Odd-Mass Nuclei}

The spectroscopy of odd-mass nuclei provides an opportunity to probe single-particle effects alongside the collective motion. Compared to eveneven nuclei where many particles contribute to the collective rotation, the odd unpaired particles can give a unique character to the different rotational bands based on single-particle excitations. Although potentially rich in information, the study and interpretation of these nuclei also presents some challenges. Firstly, the gamma-ray intensity is spread over several bands, in contrast to even-even nuclei where the yrast band concentrates most of the intensity in the decay towards the ground state. Secondly, the breaking of time-reversal symmetry leads to the fact that each collective structure based on a single-particle state consists of two stretched $E 2$ signature partner cascades linked by $\Delta I=1 \hbar$ (mixed $M 1 / E 2$ ) transitions, further fragmenting the gamma-ray intensity. In addition, particularly in heavy nuclei, the low-energy $M 1 / E 2$ transitions can be highly converted. A third complication is that often the observed rotational bands cannot be anchored to the existing level scheme or to the ground-state, due to the fact that there can be low-energy or isomeric transitions between the different band-head states. In such cases, no direct assignment of the band-head spin and parity can be made. Also, unfortunately, the moments of inertia of rotational bands based on different 
single-particle states in a given nucleus can be very similar (in the absence of particle alignment; if any it occurs generally at high rotational frequency and might not be observed). It is therefore necessary to go beyond a measurement of the rotational energy levels and exploit the properties of the nuclear electromagnetic moments. Indeed, the intensity ratio $\mathrm{T}(\Delta I=1 \hbar) / \mathrm{T}(E 2)$ is directly linked to the quadrupole electric moment $Q_{0}$ and to the orbital gyromagnetic factor $g_{K}$, or in other words to the magnetic moment $\mu$ which in general is strongly dependent on the single-particle configuration.

The radiative transition rates $\mathrm{T}_{\gamma}(E 2)$ and $\mathrm{T}_{\gamma}(M 1)$ are related to the transition energy $E$ and to the reduced transition probabilities $B(E 2)$ and $B(M 1)$ using the relations:

$$
\begin{gathered}
\mathrm{T}_{\gamma}(E 2)=1.2210^{9} B(E 2) E^{5}, \\
B(E 2, I \rightarrow I-2)=\frac{5}{16 \pi} e^{2} Q_{0}^{2}\langle I K 20 \mid(I-2) K\rangle^{2} . \\
\mathrm{T}_{\gamma}(M 1)=1.7610^{13} B(M 1) E^{3} .
\end{gathered}
$$

For rotational states and $K \neq 1 / 2$ :

$$
B(M 1, I \rightarrow I-1)=\frac{3}{4 \pi} \mu_{N}^{2}\left(g_{K}-g_{R}\right)^{2} K^{2}\langle I K 10 \mid(I-1) K\rangle^{2},
$$

with $g_{R}=Z / A$ being the rotational gyromagnetic factor and $\mu_{N}=e \hbar / 2 M c$. The static magnetic moment $\mu$ can be related to the gyromagnetic factors using

$$
\mu=\left(g_{R} I+\left(g_{K}-g_{R}\right) \frac{K^{2}}{I+1}\right) \mu_{N} .
$$

Via an intensity ratio measurement, a relevant comparison with nuclear models is possible, providing that sufficient statistics are obtained. Theoretical considerations will be discussed in section 6 ,

\subsection{Even-Z, Odd- $N$ isotopes}

In-beam spectroscopy of even- $Z$, odd- $N$ nuclei in the transfermium region has only been performed for ${ }^{253}$ No. Gamma-ray spectroscopy was first reported by Reiter et al. 21] using GAMMASPHERE and the FMA at ANL and the fusion-evaporation reaction ${ }^{207} \mathrm{~Pb}\left({ }^{48} \mathrm{Ca}, 2 n\right){ }^{253} \mathrm{No}$ with a cross-section of $\sim 500 \mathrm{nb}$. Two coincident cascades of $E 2$ transitions were observed. Since no intra-band $\Delta I=1$ transitions were observed, the structure was interpreted as being only compatible with the $\nu 7 / 2^{+}[624]$ band-head (due to the 
prediction of a small magnetic moment for this configuration). This $7 / 2^{+}$ state was otherwise interpreted as the second excited state at $379 \mathrm{keV}$ from decay spectroscopy [60]. In parallel, in-beam electron spectroscopy of ${ }^{253} \mathrm{No}$ was performed at JYFL by Herzberg et al. [20] using SACRED coupled to RITU [42]. No discrete transitions could be resolved, but cascade simulations with the gyromagnetic factor as an input parameter were fully compatible with a $\nu 9 / 2^{-}[734]$ ground-state configuration. These findings either cast doubt on the previous interpretation or signify that gamma-ray and electron spectroscopy were not highlighting the same bands due to their different electromagnetic properties. The questions were subsequently answered through detailed gamma-ray spectroscopy using JUROGAM and RITU [61, 22]. In this case, additional $\Delta I=1 \hbar$ intra-band transitions were observed linking the signature partner $E 2$ cascades with the intensity ratio $\mathrm{T}(\Delta I=1) / \mathrm{T}(E 2)$ clearly favouring assignment to the $\nu 9 / 2^{-}[734]$ ground-state band.

To date, ${ }^{253}$ No is the only odd-neutron transfermium nucleus for which inbeam spectroscopy has been successful. In the lighter transuranium isotopes, high-spin gamma-ray spectroscopy has been performed in the five isotopes ${ }^{245} \mathrm{Pu},{ }^{247,249} \mathrm{Cm}$, and ${ }^{249,251} \mathrm{Cf}$ using GAMmAsphere at ANL [7, 4, 11. Highspin states of ${ }^{249} \mathrm{Cf}$ were populated up to $\sim 28 \hbar$ using inelastic scattering while those of ${ }^{245} \mathrm{Pu}$ and ${ }^{247,249} \mathrm{Cm}$ were studied through the neutron transfer channel using ${ }^{244} \mathrm{Pu}$ and ${ }^{248} \mathrm{Cm}$ targets, respectively. Since an in-flight or other such direct identification of the nuclei produced cannot be performed, it was necessary to exploit the full resolving power of the GAMMASPHERE array in order to pin down the cascades of interest using high-fold coincidences. Two rotational bands have been observed in the three isotopes ${ }^{245} \mathrm{Pu},{ }^{247} \mathrm{Cm}$ and ${ }^{249} \mathrm{Cf}$ isotopes, up to spins of $\sim 25 \hbar$. The configurations have been assigned by comparing the $\mathrm{T}(\Delta I=1 \hbar) / \mathrm{T}(E 2)$ ratio with predictions from a Woods-Saxon model. In the three isotopes, the yrast band corresponds to the $\nu 9 / 2^{-}[734]$ configuration while bands based on single-particle excitations are interpreted as $\nu 7 / 2^{+}[624]$ in ${ }^{245} \mathrm{Pu}$ and $\nu 5 / 2^{+}[622]$ in ${ }^{247} \mathrm{Cm}$ and ${ }^{249} \mathrm{Cf}$. The yrast rotational structure is interpreted as based on the $\nu 1 / 2^{+}[620]$ Nilsson orbital in ${ }^{249} \mathrm{Cm}$ [7] and ${ }^{251} \mathrm{Cf}$ [11]. The significance and interpretation of the rotational band properties in these nuclei are discussed in section 6 .

\subsection{Even-N, Odd-Z isotopes}

As is the case for odd-neutron nuclei, there is also rather limited data available on the rotational structures in odd-proton nuclei in the region of 
heavy elements. In-beam spectroscopy of the odd-proton transfermium nuclei ${ }^{251} \mathrm{Md}$ and ${ }^{255} \mathrm{Lr}$ has been performed at JYFL using the fusion-evaporation reactions ${ }^{205} \mathrm{Tl}\left({ }^{48} \mathrm{Ca}, 2 n\right){ }^{251} \mathrm{Md}(\sigma \sim 760 \mathrm{nb})$ by Chatillon et al. [16] and ${ }^{209} \mathrm{Bi}\left({ }^{48} \mathrm{Ca}, 2 n\right){ }^{255} \operatorname{Lr}(\sigma \sim 200 \mathrm{nb}[62])$ by Ketelhut et al. [30].

In ${ }^{251} \mathrm{Md}$, a single cascade was observed with no evidence for a signature partner band. The cascade was interpreted as being a band of $E 2$ transitions based on the $\pi 1 / 2^{-}[521]$ Nilsson orbital. In this case, the $K=1 / 2$ band has a predicted decoupling parameter $a$ close to 1 , meaning that the unfavoured signature partner is shifted up in energy resulting in the observation of only a single $E 2$ cascade within the favoured band. The $1 / 2^{-}$band-head is an excited state at an energy of $55 \mathrm{keV}$ above the $\pi 7 / 2^{-}$[514] ground-state [63]. The electromagnetic properties of a possible rotational band based on this ground-state or on the predicted low-lying $\pi 7 / 2^{+}[633]$ state are not compatible with the observed sequence: see the discussion in section 6.1. It should be noted that the gamma-ray decay is dominated by the non-yrast $1 / 2^{-}$band representing only $\sim 12 \%$ of the ${ }^{251} \mathrm{Md}$ population. In a subsequent experiment, bands based on the $7 / 2^{-}$ground state and $7 / 2^{+}$excited state have been tentatively observed [64] but need to be confirmed.

The latter experiment was performed with the SAGE spectrometer allowing for combined in-beam gamma-ray and internal conversion electron spectroscopy. The data from the experiment is still under analysis, but preliminary spectra are shown in Fig. 12 to illustrate the challenge which conversion electron spectroscopy in a odd-mass transfermium nucleus represents [65]. The fusion-evaporation cross-section to produce ${ }^{251} \mathrm{Md}$ is $\sim 1 \mu \mathrm{b}$, at the limit of sensitivity for in-beam conversion electron spectroscopy.

The ground-state of ${ }^{255} \mathrm{Lr}$ corresponds to the $\pi 1 / 2^{-}[521]$ orbital and the presence of an excited state at $37 \mathrm{keV}\left(\pi 7 / 2^{-}[514]\right)$ has been deduced from decay spectroscopy [63]. It can be seen that these are the same orbitals as assigned in ${ }^{251} \mathrm{Md}$ but in the reverse order. It is therefore not surprising that a rotational band based on the $\pi 1 / 2^{-}[521]$ orbital has also been observed in ${ }^{255} \mathrm{Lr}$, with similar characteristics i.e. the absence of a signature partner. In order to disentangle the complex gamma-ray spectra it was essential to exploit the ${ }^{255} \mathrm{Lr}$ alpha decay properties. Since the $7 / 2^{-}$state is isomeric with a lifetime of $\sim 2.5$ s predominantly decaying directly to ${ }^{251} \mathrm{Md}$, tagging either on this branch or on the ground-state alpha decay could nicely reveal different gamma-ray sequences: rotational bands based on the $7 / 2^{-}$and $1 / 2^{-}$states, respectively. In agreement with the predicted electromagnetic properties of the $7 / 2^{-}[514]$ orbital, the de-excitation of the rotational band is dominated 

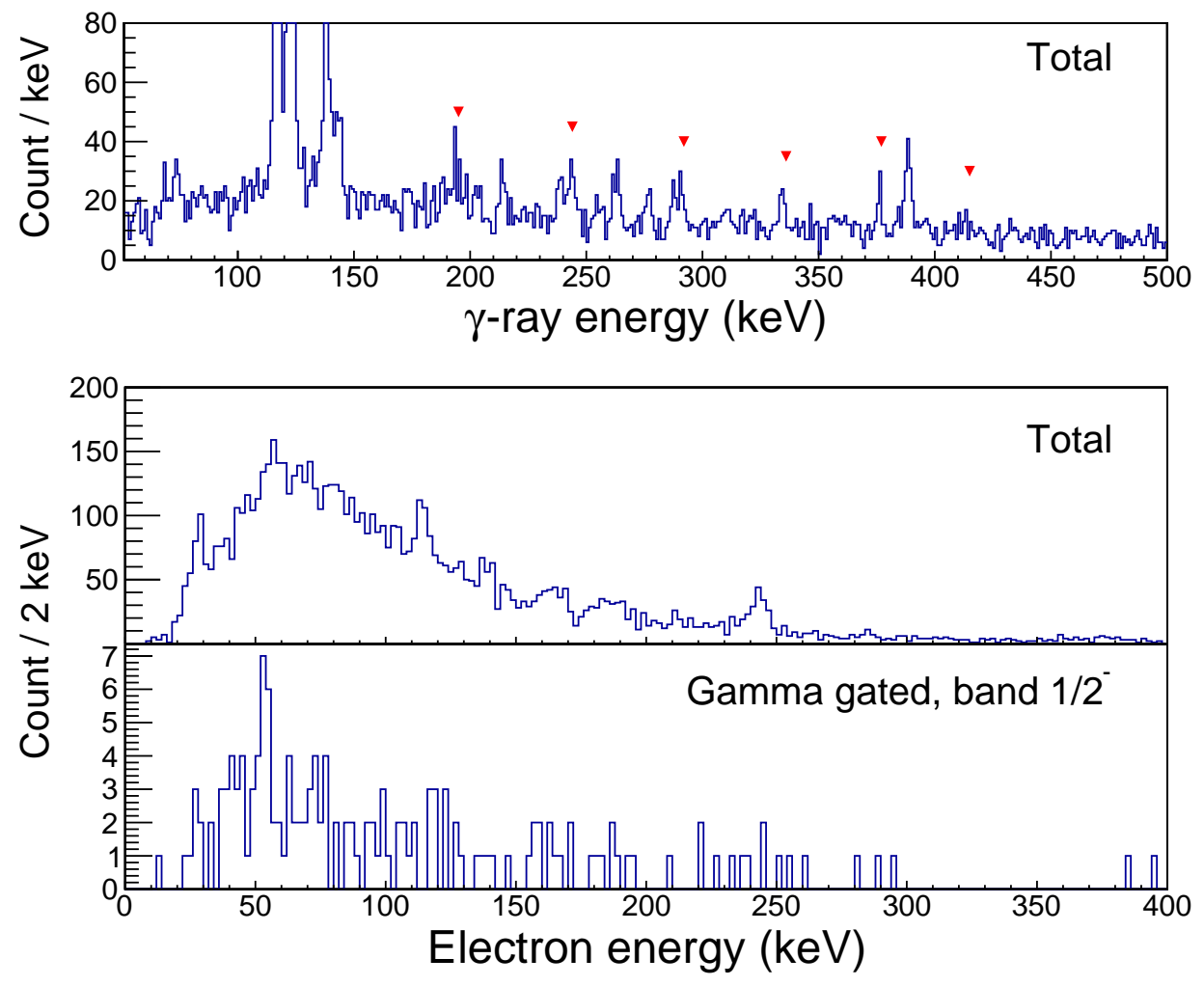

Figure 12: (Color online) Gamma-ray and conversion electron spectra of ${ }^{251} \mathrm{Md}$ obtained using the SAGE spectrometer [65. The upper and middle panels correspond to the recoilgated gamma-ray and conversion electron spectra, respectively. The bottom panel shows the spectrum of conversion electrons in coincidence with the gamma-ray transitions of the rotational band built on the $\pi 1 / 2^{-}[521]$ orbital (triangles in the top spectrum). 
by two E2 coupled sequences, although intra-band dipole transitions have not been observed which would allow confirmation of the assignment from a determination of the $T(\Delta I=1 \hbar) / T(E 2)$ intensity ratio. Exploiting the alpha-decay in the same way in ${ }^{251} \mathrm{Md}$ was not possible due to the low alpha decay branching ratio $(\sim 10 \%)$ and because no isomeric state has so far been observed in this isotope.

Rotational band structures fed from the decay of multi-quasiparticle $K$ isomeric states have been observed in ${ }^{255} \mathrm{Lr}$ by Jeppesen et al. at the Lawrence Berkeley National Laboratory [66] using decay spectroscopic techniques. One of the bands is interpreted as being built on the $\pi 9 / 2^{+}[624]$ orbital using $T(\Delta I=1 \hbar) / T(E 2)$ intensity ratio arguments, while the other is tentatively interpreted as being built on the $\pi\left(1 / 2^{-}[521] \otimes 7 / 2^{-}[514] \otimes 9 / 2^{+}[624]\right)$ three quasiparticle isomeric state. It is interesting to note here the nice complementarity between the in-beam and decay spectroscopic techniques which enhance different parts of the level scheme. Similar results have also been obtained in experiments carried out in JINR, Dubna and at GSI, Germany [67, 68]. In the former study of Hauschild et al., it is concluded that the isomeric state is likely to be formed by coupling the valence proton to a two neutron quasiparticle state, as opposed to the conclusion of Jeppesen et al. mentioned above.

Considering the lightest isotopes in the region, only two nuclei have been studied to high-spins using inelastic scattering on ${ }^{237} \mathrm{~Np}$ and ${ }^{241} \mathrm{Am}$ targets [2]. The experimental technique was similar to that used to study the odd-neutron nuclei ${ }^{245} \mathrm{Pu},{ }^{247,249} \mathrm{Cm}$ and ${ }^{249} \mathrm{Cf}$ [4, 7]. In ${ }^{237} \mathrm{~Np}$, two rotational band structures have been observed and interpreted as $\pi 5 / 2^{+}[642]$ (intruder $\pi \mathrm{i}_{13 / 2}$ orbital) and $\pi 5 / 2^{+}[523]$ configurations. Rotational structures based on the same orbital are also observed in ${ }^{241} \mathrm{Am}$, together with a third rotational band based on the $\pi 3 / 2^{-}[521]$ configuration. These studies allow the effect of high- $j$ orbitals and of the pairing correlations to be tracked, aspects which are discussed further below in section 6 .

\section{Isomeric high- $K$ two quasiparticle states}

In a similar manner to the study of odd-mass isotopes discussed above, the study of two quasiparticle states in deformed even-even nuclei can reveal information on the single-particle configurations and on the pair gap. An 
expression for the band-head energies of the two quasiparticle state is given by:

$$
E_{2 q p}=E_{1}^{q p}+E_{2}^{q p}=\sqrt{\left(\epsilon_{1}-\lambda\right)^{2}+\Delta^{2}}+\sqrt{\left(\epsilon_{2}-\lambda\right)^{2}+\Delta^{2}}
$$

where $\epsilon_{\nu}$ is the single-particle energy, $\lambda$ the Fermi energy and $\Delta$ is the pairgap energy. As noted in the review of Kondev et al. 69, the most important orbitals for the construction of high- $K$ states in the $A \simeq 250$ region are the proton $5 / 2^{-}[512], 7 / 2^{+}[633], 7 / 2^{-}[514]$ and $9 / 2^{+}[624]$ and the neutron $5 / 2^{+}[622], 7 / 2^{+}[624], 7 / 2^{+}[613]$ and $9 / 2^{+}[734]$ : see the single-particle energy diagrams in Fig. 13 .

The decay of these states may involve a large change in $K$ value, leading to a significant lifetime. In general the lifetime can be related to the degree of forbiddenness $\nu=|\Delta K-\lambda|$ where $\Delta K$ is the difference in $K$ value of the initial and final states and $\lambda$ is the multipole order of the transition. The systematics of the lifetimes for particular transition multipolarities have been tabulated, for example by Löbner [72]. Löbner showed that the hindrance roughly increases by a factor of 100 for each added degree of forbiddenness. However, many exceptions to this rule-of-thumb can be found in the literature (see Kondev et al. for a discussion [69]).

An experimental determination of the spin and parity $\left(K^{\pi}\right.$-value) of the isomeric state provides a strong indication of the single-particle states involved, as relatively few combinations of particles can give rise to high- $K$ states. The isomeric nature of the high- $K$ states means that they can be relatively easily isolated in experiments, and the relation to the underlying single-particle spectrum provides an excellent tool for comparison of experiment with theory. Another consequence of $K$-isomerism has been pointed-out by $\mathrm{Xu}$ et al. [73]: the blocking effect of the unpaired nucleons reduces the pairing and increases the fission barrier height therefore resulting in an increased survival probability. As in the case of odd-mass nuclei, the rotational band characteristics (signature splitting, $\mathrm{T}(\Delta I=1 \hbar, M 1+E 2) / \mathrm{T}(E 2))$ are dependent on the quasiparticles involved in the high- $K$ configuration. The observation and investigation of the rotational bands feeding high- $K$ states can therefore aid configuration assignments. Again, the breaking of a pair of nucleons and re-coupling in a two quasiparticle state strongly reduces pairing correlations due to blocking, which therefore increases the moment of inertia as compared to the yrast states in even-even nuclei. It is important to stress that two quasiparticle states are highly non-yrast due to the fact that a pair 


\section{a) Woods-Saxon}

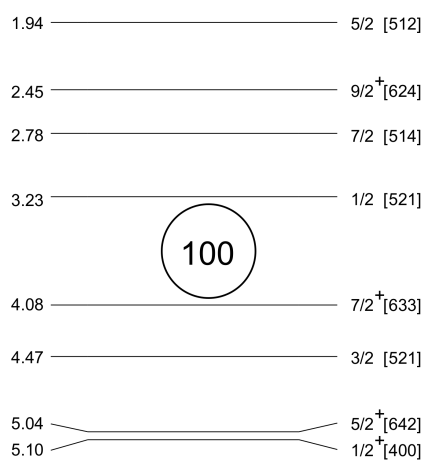

b) HFB + D1S

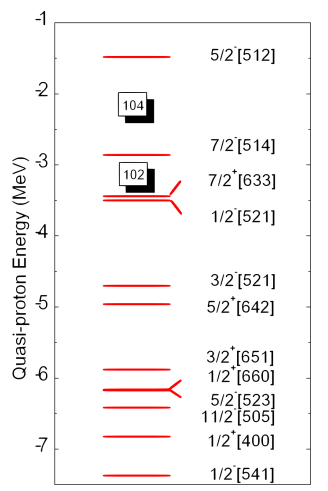

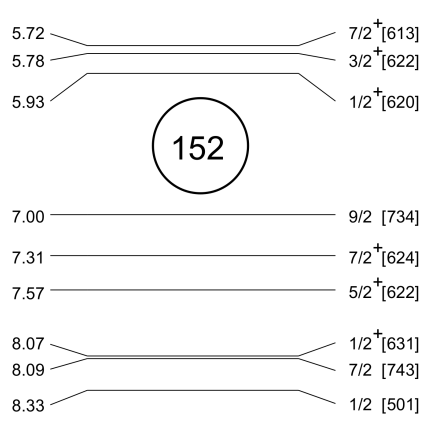

c) HFB + Sly4

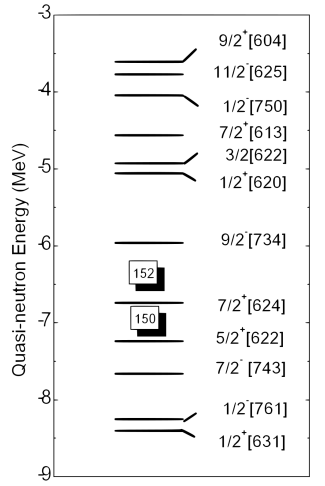

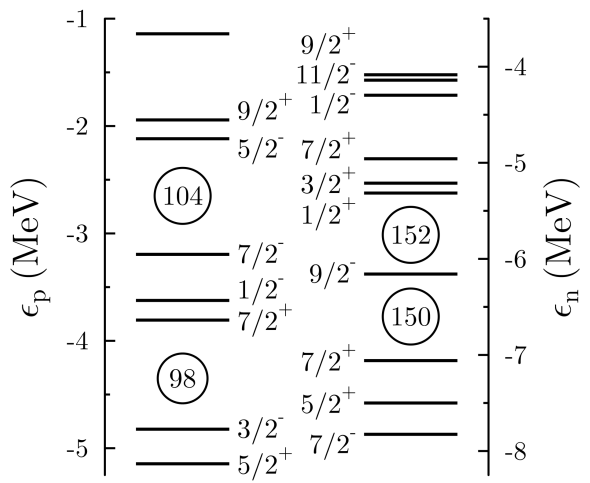

Figure 13: Single particle energies in ${ }^{250} \mathrm{Fm}$ using different formalisms a) WoodsSaxon [13, b) HFB+Gogny D1S [70, c) HFB+Sly4 [71. For each panel the proton (neutrons) energies are drawn on the left (right). The single-particles are labelled using their asymptotic Nilsson label in panels a) and b), spin and parity in panel c). 
has to be broken to create the state. The price to pay in energy to break a pair is related to the pair-gap energy which approximates to $\Delta \sim 12 / A^{1 / 2}$ $\mathrm{MeV}$. The excitation energy of a two quasiparticle state is roughly $1 \mathrm{MeV}$ in the $A \sim 250$ region. Should the excitation occur across the deformed shell gaps at $Z=100$ or $N=152$, additional energy is required.

The limited number of combinations of single-particle states which can give rise to high- $K$ states explains why only a few rotational bands based on these states are known so far in the $\mathrm{A} \sim 250$ mass region. On the theoretical side, a description of the two quasiparticle states and associated rotational bands is also a challenge. In addition to accurate single-particle energies, a proper theoretical description includes time-reversal symmetry breaking, and blocking effects. Additional complications occur at high excitation energies due to the onset of other excitation modes such as vibrations, octupole correlations, and so on, whose inclusion is even more challenging.

\subsection{Experimental studies}

Long-lived two quasiparticle isomeric states were first reported in the $A \sim 250$ mass region more than forty years ago by Ghiorso et al. in ${ }^{250} \mathrm{Fm}$ and ${ }^{254}$ No [74]. However, the experimental apparatus did not allow the excitation energy or decay path to be resolved, though the authors provided speculation as to the configuration of the isomeric states. In later years, decay spectroscopic techniques were used to study two quasiparticle isomeric states in the actinide region: see table 2. The table is not exhaustive, see also the review of Kondev et al. [69] and the tabulation of isomeric states above $Z=82$ compiled by Herzberg and Cox [75] for more complete listings.

Among these cases, rotational bands built on the high- $K$ isomeric states have only been observed in the isotopes ${ }^{250} \mathrm{Fm}$ [15] and ${ }^{252} \mathrm{No}$ [19], both studied at JYFL: see the partial level schemes shown Fig. 14. The experimental technique was similar in both cases and follows the life of the nucleus through its decay path after production in a fusion-evaporation reaction: (i) prompt cascade of gamma-rays emitted from excited states (ii) implantation at focal plane of recoil separator (iii) isomer decay (iv) alpha decay. Observation and correlation (spatially and temporally) of the radiation emitted in each step forms the basis of the recoil-decay tagging (RDT) technique described earlier. In this case, the sequence differs from the usual RDT technique by step (iii), resulting in the gamma-ray spectra displayed in Fig. 15. The experimental observation of high- $K$ rotational bands represents a challenge given 


\begin{tabular}{|l|l|l|l|l|}
\hline Nucleus & $K^{\pi}$ & $\mathrm{E}(\mathrm{keV})$ & $t_{1 / 2}$ & Reference \\
\hline${ }^{244} \mathrm{Pu}$ & $8^{-}$ & 1216 & $1.8(2) \mathrm{s}$ & {$[76]$} \\
\hline${ }^{244} \mathrm{Cm}$ & $6^{+}$ & 1040 & $34 \mathrm{~ms}$ & {$[77]$} \\
\hline${ }^{246} \mathrm{Cm}$ & $8^{-}$ & 1179.7 & $1.12(24)$ & {$[78,[79]$} \\
\hline${ }^{248} \mathrm{Cm}$ & $8^{-}$ & 1409 & $146(18) \mu \mathrm{s}$ & {$[78,[80]$} \\
\hline${ }^{248} \mathrm{Cf}$ & $8^{-}$ & $1261(2)$ & & {$[81]$} \\
\hline${ }^{250} \mathrm{Fm}$ & $8^{-}$ & 1195 & $1,92(5) \mathrm{s}$ & {$[15]$} \\
\hline${ }^{256} \mathrm{Fm}$ & $7^{-}$ & 1425.5 & $70(5) \mathrm{ns}$ & {$[82]$} \\
\hline${ }^{250} \mathrm{No}$ & $6^{+}$ & & $43{ }_{-15}^{+22} \mu \mathrm{s}$ & {$[83]$} \\
\hline${ }^{252} \mathrm{No}$ & $8^{-}$ & 1255 & $109(6) \mathrm{ms}$ & {$[79]$} \\
& & 1254 & $109(3) \mathrm{ms}$ & {$[19]$} \\
\hline \multirow{2}{*}{${ }^{254} \mathrm{No}$} & $8^{-}$ & 1293 & $266(2) \mathrm{ms}$ & {$[84]$} \\
& & 1296 & $266(10) \mathrm{ms}$ & {$[85]$} \\
& & $1295(2)$ & $275(7) \mathrm{ms}$ & {$[86]$} \\
\hline \multirow{2}{*}{${ }^{256} \mathrm{R} \mathfrak{j}$} & $(6,7)$ & $\sim 1120$ & $263(2) \mathrm{ms}$ & {$[87]$} \\
\hline${ }^{270} \mathrm{Ds}$ & $(10-12)$ & $\sim 1400$ & $17(2) \mu \mathrm{s}$ & {$[88]$} \\
& & & $17-23 \mu \mathrm{s}$ & {$[89]$} \\
\hline
\end{tabular}

Table 2: Two quasiparticle isomeric states in the region of heavy elements.

${ }^{a}$ In ${ }^{256} \mathrm{Rf}$ Robinson et al. observe a $17(5) \mu$ s isomer interpreted as a 4qp excitation 91 . 
the low transition energies, fragmentation of decay intensity over many transitions and the fusion-evaporation cross-sections which are of $\sim 1 \mu \mathrm{b}$ and $\sim 200 \mathrm{nb}$ in the case of ${ }^{250} \mathrm{Fm}$ and ${ }^{252} \mathrm{No}$, respectively. The decay of the high- $K$ isomeric state usually proceeds through an intermediate rotational band structure ( $K^{\pi}=2^{-}$in the case of ${ }^{250} \mathrm{Fm}$ and ${ }^{252} \mathrm{No}$ as shown in Fig. 14) in which, due to the very low energies, the transitions are highly converted. Therefore, the decay of the isomeric state of the implanted nucleus deposits a reasonable amount of energy in the implantation detector corresponding to conversion electrons, Auger electrons and x-rays. The signal from the decay of the isomeric state can be exploited in the data analysis (step (iii) above). This so-called calorimetric technique was suggested by Jones [92] and pioneered in the transfermium region $\left({ }^{254} \mathrm{No}\right)$ in parallel works by Herzberg et al. 84] at the JYFL and Tandel et al. [85] at ANL.

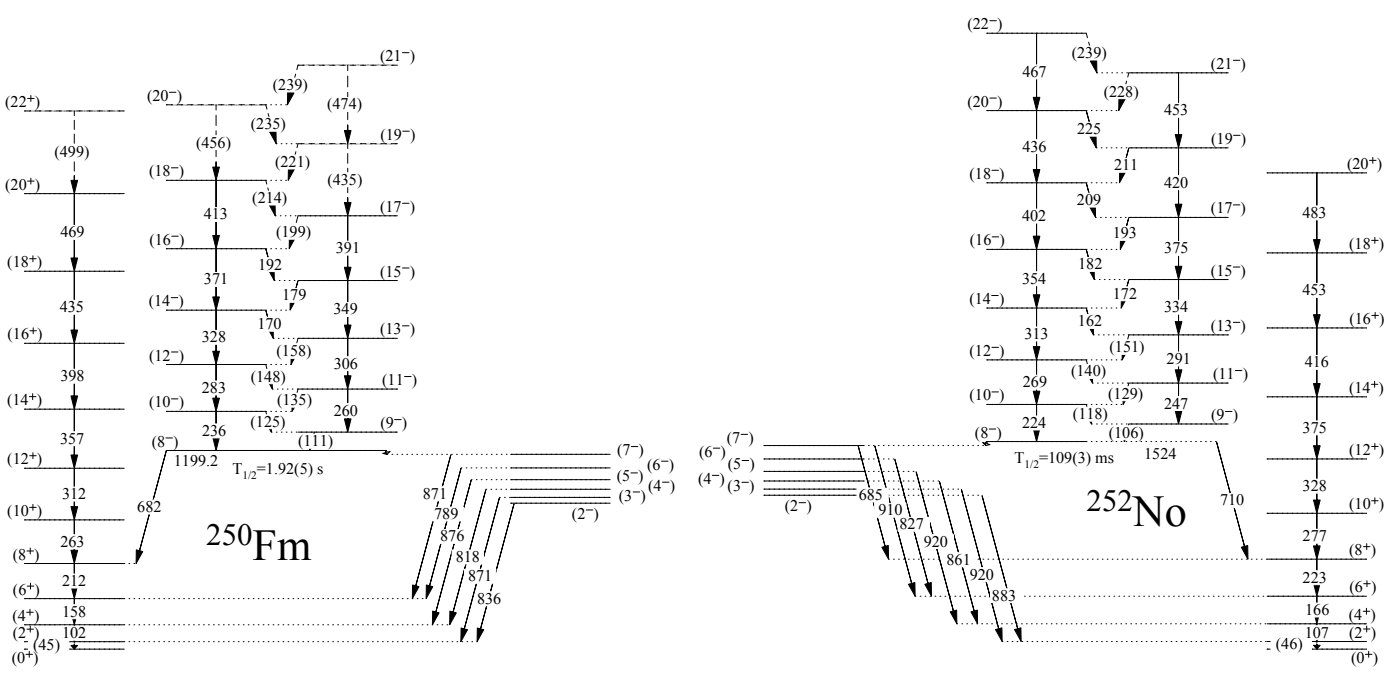

Figure 14: Partial level schemes of ${ }^{250} \mathrm{Fm}$ and ${ }^{252} \mathrm{No}$, adapted from [15, 19]. The intraband transitions in the $K^{\pi}=2^{-}$band are not shown.

The decay scheme of the isomeric state provides an initial basis for the two quasiparticle configuration assignment as it usually provides the spin, parity and excitation energy of the high- $K$ state. A comparison with theory and the expectations from the orbitals listed above which can lead to high- $K$ states strongly constrains the assignment but this is not unambiguous. A case in point is the $8^{-}$isomeric state in ${ }^{254} \mathrm{No}$, where different interpretations of rather similar data obtained in several decay spectroscopy experiments have been presented. While Herzberg et al. [84], Tandel et al. [85] and Heßberger 


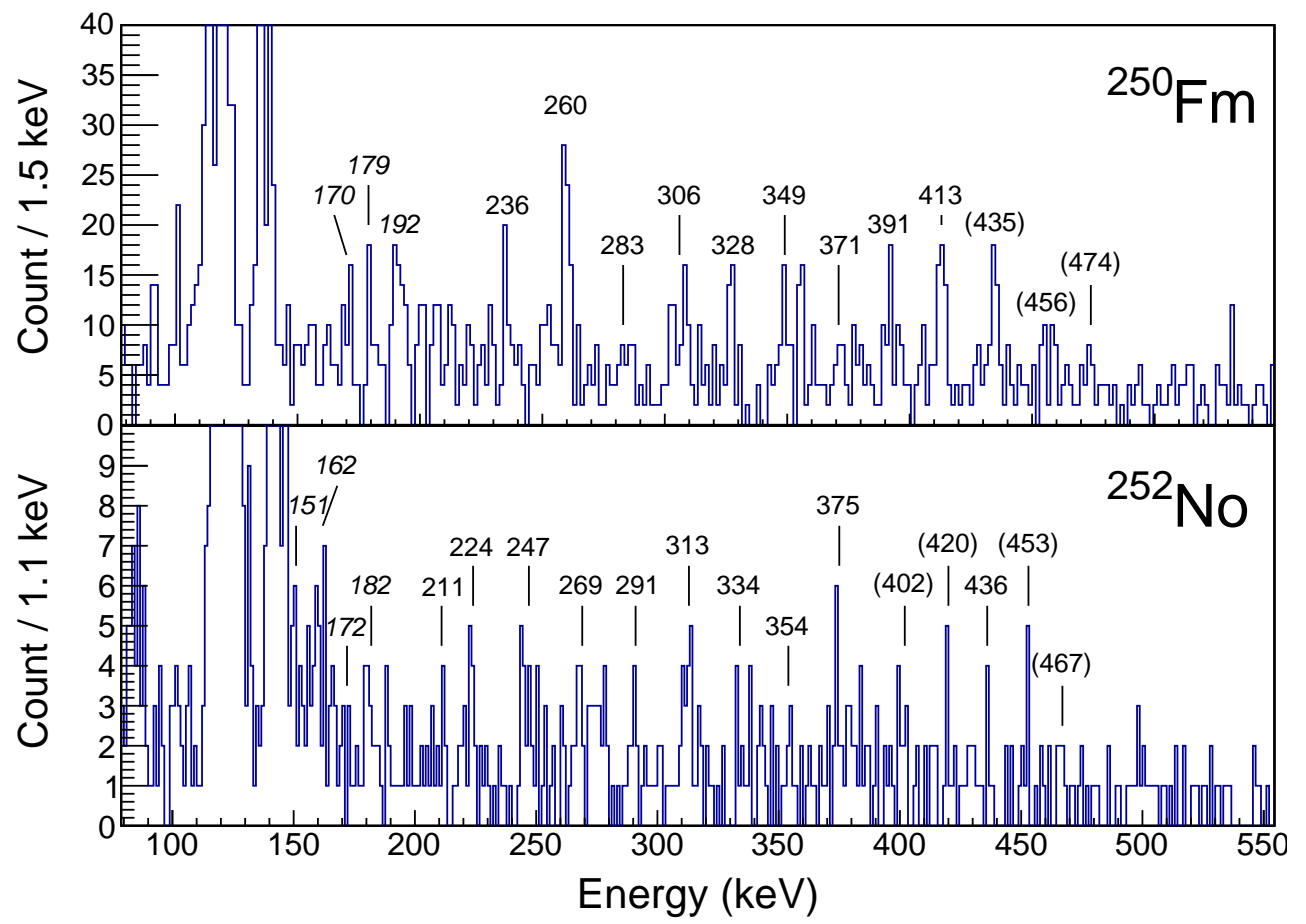

Figure 15: Singles gamma-ray spectra in ${ }^{250} \mathrm{Fm}$ and ${ }^{252}$ No tagged with the decay of an isomeric state obtained using the RITU/JUROGAM/GREAT set-up in JYFL [19, 15]. The rotational bands are built on a $K^{\pi}=8^{-}$isomeric state. See also the corresponding level schemes shown in Fig. 14 
et al. [86] assign a $\pi 7 / 2^{-}[514] \otimes \pi 9 / 2^{+}[624]$ configuration, Clark et al. 87] suggest conversely a $\nu 7 / 2^{+}[613] \otimes \nu 9 / 2^{-}[734]$ configuration, with additional differences in the interpretation of the other states observed.

In order to have an unambiguous interpretation, combined in-beam and decay spectroscopic studies are essential since, as in the case of odd-mass isotopes discussed previously, the rotational bands can provide a configuration assignment through a measurement of the $T(\Delta I=1 \hbar) / T(E 2)$ intensity ratio. In most cases, the population of the high- $K$ band is much weaker than the ground-state rotational band and can only be observed by the use of the highly selective isomer tagging technique discussed above.

In both ${ }^{250} \mathrm{Fm}$ and ${ }^{252} \mathrm{No}$, the rotational bands built on the high- $K$ isomeric state display moments of inertia with common features as shown in Fig. 16. The $\mathcal{J}^{(1)}$ kinematic moment of inertia is larger than that of the ground-state band, which is a result of the blocking which reduces the pairing correlations. It is also flatter (more constant) as a function of the rotational frequency compared to the yrast ground-state band. While this behaviour is characteristic of rotations built on two quasiparticle states, it does not constrain the configuration assignment as the moments of inertia for the different possible two quasiparticle configurations are expected to be similar (for example two-neutron or two-proton $8^{-}$states). Another common feature of ${ }^{250} \mathrm{Fm}$ and ${ }^{252} \mathrm{No}$, related to the behaviour of the dynamical moment of inertia $\mathcal{J}^{(2)}$, is discussed in section 6.2 .

While the spin and parity of the band-bead is deduced (from decay spectroscopy), the remaining question is often whether the two quasiparticle excitation is due to neutrons or protons. A firmer assignment can be provided by the electromagnetic properties, namely the comparison of the experimental intensity ratio $T(\Delta I=1 \hbar) / T(E 2)$ with theoretical predictions. Indeed the orbital and spin gyromagnetic factors ( $g_{s}$ and $g_{l}$ respectively) of neutrons and protons are strongly different. In the particular case of singlet states $(\Sigma=0)$ which are energetically favoured according to the Gallagher rules [93], the spin gyromagnetic factors cancel and the remaining contribution is mainly from the angular momentum of the nucleons [94]: $g_{K}=g_{l}^{p(n)}=1(0)$. As an example, the $g_{K}$ factors inferred from Woods-Saxon calculations are 0.01 for the $\nu 7 / 2^{+}[613] \otimes \nu 9 / 2^{-}[734]$ coupling and 1.01 for the $\pi 7 / 2^{-}[514] \otimes \pi 9 / 2^{+}[624]$ for the possible $8^{-}$states in ${ }^{252} \mathrm{No}\left[19\right.$. In both ${ }^{250} \mathrm{Fm}$ and ${ }^{252} \mathrm{No}$ (and indeed across the $N=150$ isotones), the neutron two quasiparticle configuration $\nu 7 / 2^{+}[613] \otimes \nu 9 / 2^{-}[734]$ configuration has been established [15, 19].

In the case of low statistics, discrete $\Delta I=1 \hbar$ transitions may not be 
resolved in the gamma-ray spectra. Parr et al. [95] developed an alternative analytical method which compares the expected number of events for $\Delta I=1 \hbar$ transitions, assuming different configurations, to the statistics in the region of the spectrum where they are expected to be found. The technique was successfully applied to the known cases of ${ }^{250} \mathrm{Fm}$ and ${ }^{252} \mathrm{No}$.

Transfer reactions (where a suitable target is available) can also provide an unambiguous configuration assignment, indicating proton, neutron or mixed constituents [94. In addition, theses reactions populate only two quasiparticle states where one of the quasiparticles constitutes the groundstate of the odd-A target. Beta decay can also provide a configuration indicator. Examples of configuration assignments from transfer reaction and beta decay in the $N=150$ isotones are discussed by Robinson et al. [79].

Besides the ${ }^{250} \mathrm{Fm}$ and ${ }^{252} \mathrm{No}$ cases, evidence for a rotational band built on a two quasiparticle configuration has been found in ${ }^{244} \mathrm{Pu}$ [76]. As only in-beam spectroscopy was performed, it was not possible to correlate the band with the decay of an isomeric state.

It should be remembered that the first evidence for non-yrast states in the transfermium region from in-beam studies were found in ${ }^{254}$ No by Eeckhaudt et al. at JYFL [28]. Transitions linking a tentatively assigned $3^{+}$two quasiparticle state to the ground-state band were observed and later confirmed by decay spectroscopic techniques. It is interesting to note that this work certainly triggered further studies of non-yrast states in the nobelium region, which were successful in ${ }^{250} \mathrm{Fm}$ and ${ }^{252} \mathrm{No}$, but which are surprisingly still not fully completed in ${ }^{254}$ No. Although ${ }^{254}$ No has the largest production cross section in this region and despite the fact that a number of in-beam spectroscopic studies have focussed on it, it has not been yet possible to observe a rotational band built on the known $K^{\pi}=8^{-}$two quasiparticle isomer. Exclusively from decay studies, evidence for rotational states built on this isomeric state (fed by the decay of another higher lying isomeric state) have been reported by Heßberger et al. using SHIP at GSI [86] and Clark et al. using the Berkeley Gas-Filled Separator [87]. As stated above, however, the level scheme and interpretation of these two collaborations differ. It is clear that further detailed in-beam and decay studies of ${ }^{254} \mathrm{No}$ are required to clarify the nature of the $K^{\pi}=8^{-}$isomer and the associated high-spin rotational band. 


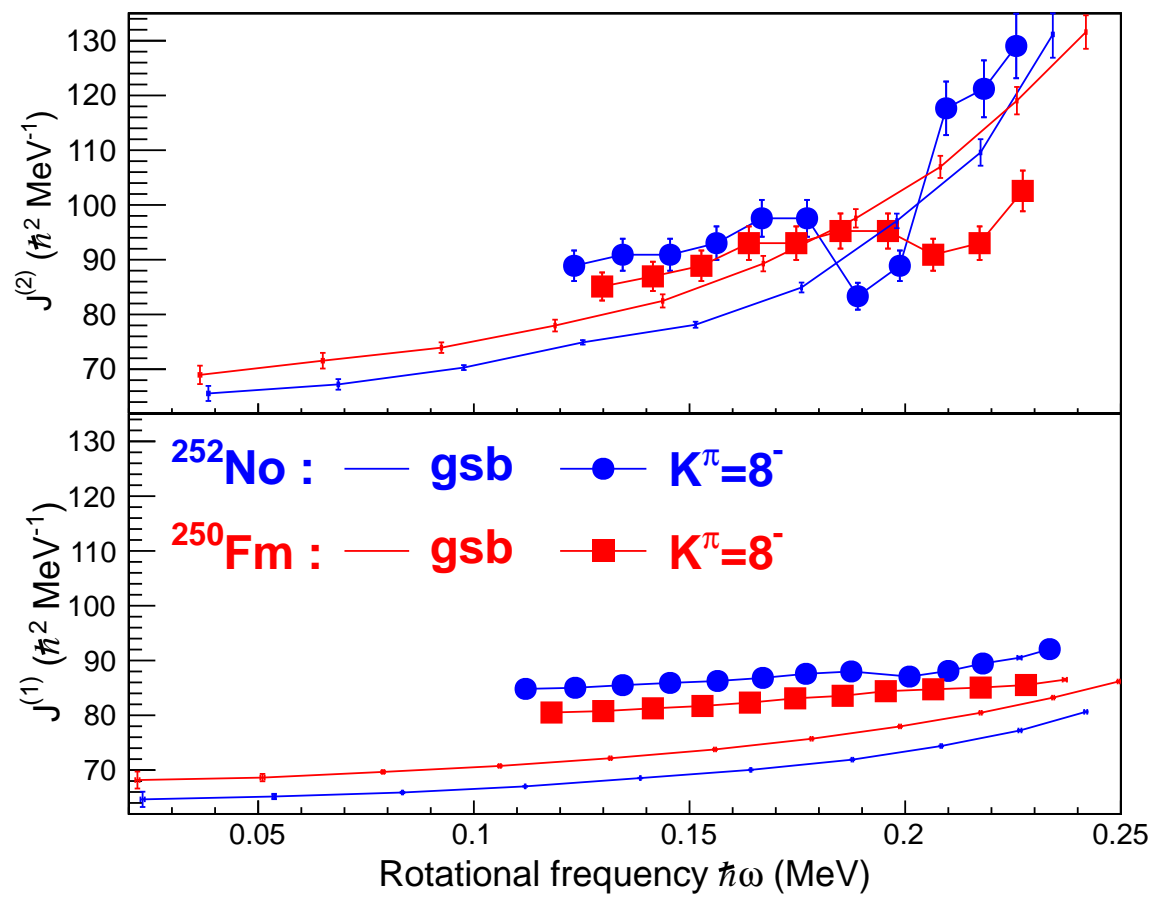

Figure 16: (Color online) Dynamic (top panel) and kinematic (low panel) moment of inertia for the ground state band (gsb) and high- $K$ band of ${ }^{250} \mathrm{Fm}$ (red) and ${ }^{252} \mathrm{No}$ (blue). Curves for the ground-state bands are displayed without markers. Red squares (blue circles) correspond to the high- $K$ band of ${ }^{250} \mathrm{Fm}\left({ }^{252} \mathrm{No}\right)$. Adapted from [19]. 


\section{Discussion}

It is well-known that the shell correction energies and boundaries of the regions of the largest shell corrections are governed by the locations of the high- $j$ orbitals at sphericity, for example the neutron $h_{11 / 2}, k_{17 / 2}$ and $j_{13 / 2}$ orbitals in the region of $N=184$ and the proton $\mathrm{i}_{13 / 2}, \mathrm{i}_{11 / 2}$ and $\mathrm{j}_{15 / 2}$ orbitals in the region of $Z=114-126$ (see e.g. Bender et al. and references therein [47]). In addition, the spin-orbit partners $2 \mathrm{f}_{5 / 2}$ and $2 \mathrm{f}_{7 / 2}$ are of particular interest since they determine the existence of the $Z=114$ and/or $Z=120$ proton spherical gaps. It is therefore of importance to try to investigate and locate the positions of the high- $j$ states, as these have a significant effect on the shell corrections of superheavy nuclei. The locations of high- $j$ orbitals in lighter nuclei can also be used to benchmark theoretical predictions. If the theories can reproduce the experimental data in lighter nuclei, then the predictions of the properties of superheavy nuclei will be on a firmer footing. The locations of single-particle states in deformed nuclei are sensitive to the location of individual parent orbitals at sphericity, therefore any disparity in deformed nuclei reflects disparities at sphericity.

The observation of rotational bands based on the $\left(2 \mathrm{f}_{5 / 2}\right) \pi 1 / 2^{-}[521]$ orbital in ${ }^{251} \mathrm{Md}$ and ${ }^{255} \mathrm{Lr}$ is therefore an interesting benchmark in the transfermium region. Rotational bands based on the same orbital were previously observed in ${ }^{247} \mathrm{Bk}$ and ${ }^{251}$ Es using decay spectroscopy techniques [96, 97] but only at the lowest angular momenta. The study of high- $j$ intruder orbitals such as $\nu 9 / 2^{-}[734]\left(\nu \mathrm{j}_{15 / 2}\right)$ or $\pi 9 / 2^{+}[624], \pi 7 / 2^{+}[633], \pi 5 / 2^{+}[642]\left(\pi \mathrm{i}_{13 / 2}\right)$ is also relevant since their positions are not necessarily well known. As mentioned above, several of these orbitals are responsible for generating the high$K$ states in the nobelium region, thus can be accessed through the study of such isomeric states. In addition, the neutron $\mathrm{j}_{15 / 2}$ and proton $\mathrm{i}_{13 / 2}$ orbitals are responsible for the rotational alignment effects observed in the region. These orbitals attract great interest not only for their relevance in the (super)heavy region, but also because they are active in the SD bands of the A 190 region as super-intruder orbitals responsible for the global behaviour of the moment of inertia. We have here therefore a unique opportunity to test the influence of these high- $j$ orbitals (effect of alignment on the moment of inertia) and of pairing correlations in different rotation and deformation regimes. In the following, the relevance of the experimental observables is discussed in parallel with a summary of the findings of some of the recent theoretical works which have been published. 


\subsection{Moments of inertia and rotational properties}

The dynamical moment of inertia $\left(\mathcal{J}^{(2)}\right)$ has the advantage that it is independent of any spin assumption, for example on any speculated single-particle configuration. Unfortunately, the $\mathcal{J}^{(2)}$ for $n$-quasiparticle band-head configurations are usually similar, which does not provide a tool for assignment (the particular case of back- and up- bending will be discussed below). The kinematical moment of inertia $\left(\mathcal{J}^{(1)}\right)$ is more sensitive to the single-particle structure. However, comparisons with theory or configuration assignments made by comparing experimental data with theoretical calculations have to be taken with care. Indeed, the spin has to be known to calculate the experimental $\mathcal{J}^{(1)}$, which can only be done assuming the spin of the band-head. Changing the band-head spin hypothesis changes also $\mathcal{J}^{(1)}$, which can accidentally match with theoretical predictions even if the configuration assignment is wrong. A typical example here is the case of ${ }^{253}$ No: assuming either the $\nu 7 / 2^{+}[624]$ or $\nu 9 / 2^{-}[734]$ band-head leads to different $\mathcal{J}^{(1)}$ that in both cases are well reproduced by theory: see e.g. [98], Fig. 2 or [99], Fig. 19. For similar reasons, the "Harris" technique [100, 101] often used to assign the spins of rotational bands can lead to non-unique solutions when applied to odd-mass nuclei: see also the discussion of the method in [102, 103].

A large number of theoretical works have been dedicated to reproduction of the systematic behaviour of the moments of inertia in this region. A common finding of many studies (in even-even as well as in odd nuclei) is that the pairing strength needs to be decreased by $10-15 \%$ to reproduce the experimental data: see cranked Woods-Saxon calculations by Abu Saleem et al. [2, Tandel et al. [7. Afanasjev et al. [104, 105] also reduced the BrinkBooker part of the Gogny D1S force in the pairing channel to reproduce the moment of inertia of VHE nuclei. In this detailed study, it turns out that a reduction of pairing is corroborated by $\Delta^{(3)}$ three-point mass indicator and that pairing might be slightly weaker for the lighter actinides. On the other hand, moments of inertia are well reproduced in actinide nuclei in the extensive study of Delaroche et al. using HFB calculations with the D1S Gogny interaction [106]. Using cranked HFB calculations, Duguet et al. [107] also tested different surface or volume pairing parameterisations. Using a cranked shell model with particle-number conserving method calculations, He et al. [98] and Zhang et al. [99, 108, have adjusted the pairing strength to fit with the experimental $\mathcal{J}^{(1)}$ of ${ }^{253}$ No or to the experimental odd-even mass differences. The $(\kappa, \mu)$ Nilsson parameters were also re-adjusted to better reproduce the single-particle spectrum. Pairing is also adjusted in [109] to 
reproduce the energy of the $2^{+}$rotational state. In contrast, in the work of Shi et al. using Skyrme-based density functional theory, it was necessary to increase the pairing strength in order to reproduce the experimental value of the kinematical moment of inertia $\mathcal{J}^{(1)}$ in ${ }^{252}$ No [110]. It was also not possible to simultaneously reproduce the moment of inertia and the odd-even mass staggering. The adjustment of pairing in the theoretical models is thus still an open question.

In general, the various theoretical approaches give a reasonable description of the moments of inertia, but discrepancies appear under close inspection. Often the behaviour as a function of rotational frequency (alignment) is well-reproduced, but the trends and subtle differences as a function of nucleon number are not fully under control. For example, in the work of Shi et al. [110] the reproduction of the moment of inertia of ${ }^{252}$ No is excellent (the pairing is adjusted here), but the agreement is not as good as the proton or neutron number is changed.

Another observable is the rotational alignment, which is the total angular momentum minus a smooth fitted reference e.g. at the lowest frequencies of a neighbouring even-even reference nucleus in the case of rotational bands in odd-mass nuclei. In the A 250 mass region the alignment is due, as mentioned above, to the high- $j$ orbitals $\pi \mathrm{i}_{13 / 2}$ and $\nu \mathrm{j}_{15 / 2}$ : see [2, 7, 99, 104, 105, 106, 108, 107, 111, 112, 113, 114, 115], etc. It is also important to note that the alignment is sensitive to the pairing strength: see e.g. the analysis in [2, 7]. In odd-mass nuclei, the blocking of a particular state by the odd particle imposes some restrictions on the particle responsible for deviations from a smooth behaviour: the filling of specific orbitals can either allow or block the alignment. The discussion is however rather limited in the odd-mass transfermium nuclei since no significant alignment effects have yet been observed, likely because the alignments occur above the experimentally observed rotational frequencies.

In the lightest odd- $Z{ }^{241} \mathrm{Am}$, signs of an up-bending at the highest observed rotational frequencies [2] are interpreted as a gradual alignment of the $\nu \mathrm{j}_{15 / 2}$ orbital. From several studies, it seems conversely that the $\nu \mathrm{j}_{15 / 2}$ intruder orbital does not play a major role in the sharp backbending of even-even actinide isotopes see e.g. [2, 99, 105, 108, 113]. Recent systematic studies in the lightest $N=151$ plutonium, curium and californium isotopes up to $\approx 25 \hbar$ using radioactive targets and $\mathrm{Pb}$ and $\mathrm{Bi}$ beams at GAMMASPHERE have been able to shed some light on this long-standing puzzle [4]. Two bands each, one built on the ground state $\left(\nu 9 / 2^{-}[734]\right)$ and the other on an excited 

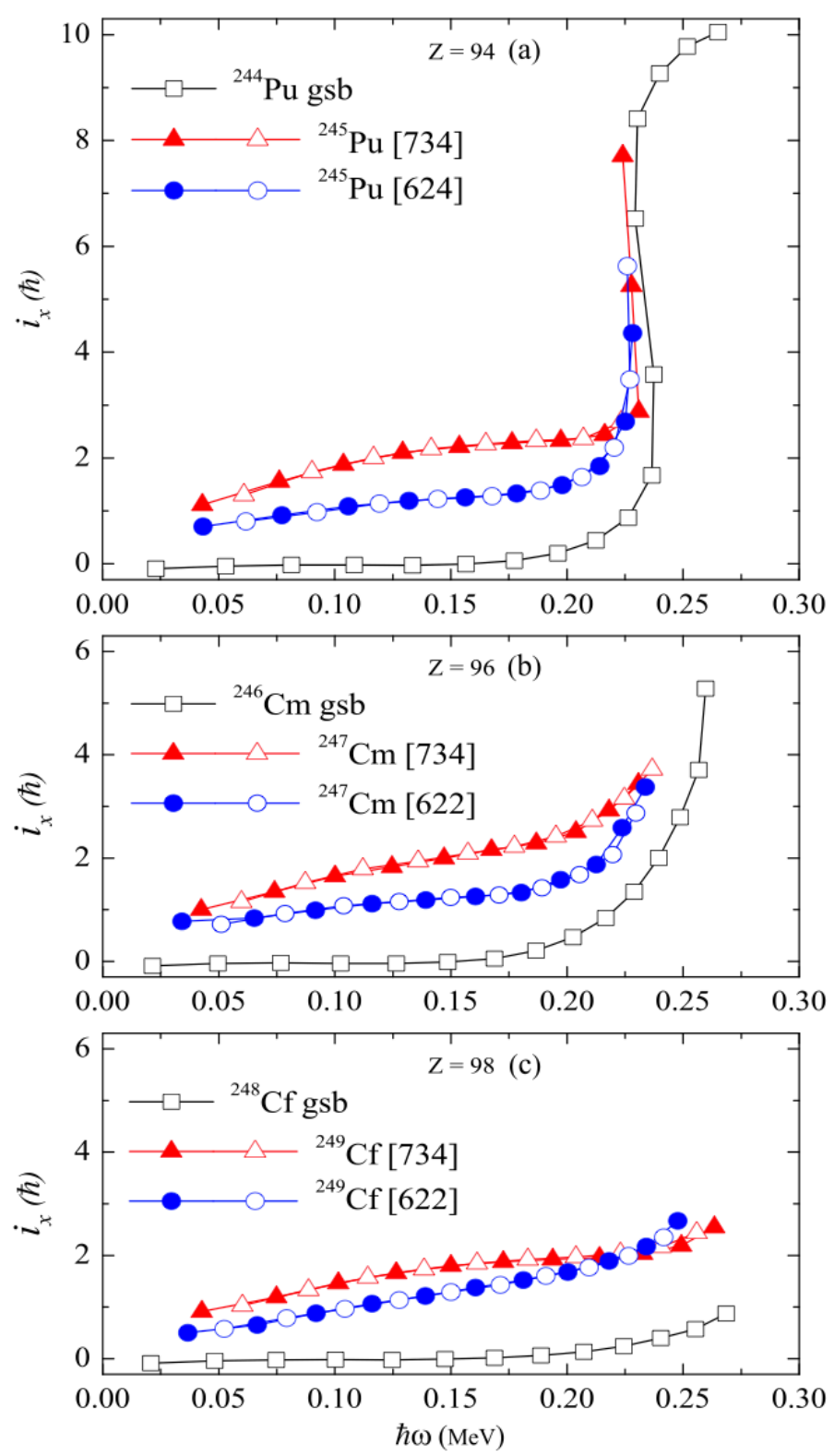

Figure 17: (Color online) Experimental alignment for the new bands observed in (a) ${ }^{245} \mathrm{Pu}$, (b) ${ }^{247} \mathrm{Cm}$ and (c) ${ }^{249} \mathrm{Cf}$, compared with ground-state bands of even-even neighbours. Taken from Hota et al. [4, Creative Commons Attribution (http://creativecommons. org/licenses/by/3.0/). 
state, have been identified in a series of $N=151$ nuclei with $94 \leq Z \leq 98$. Since the ground state band in each isotone is built on a $j_{15 / 2}$ neutron orbital, and the excited band is not, this allows a systematic comparison of the alignments in the different bands with their respective even-even cores, where the $\mathrm{j}_{15 / 2}$ neutron alignment is blocked in the ground state band but not in the excited band. The analysis therefore provides experimental proof that the alignment in at least the ${ }^{245} \mathrm{Pu}$ yrast band involves $\mathrm{i}_{13 / 2}$ protons: see Fig. 17. With the same orbital configuration, only the onset of an up-bend at the highest observed spin is seen in the yrast band of ${ }^{247} \mathrm{Cm}$, while it is not observed in the yrast bands of ${ }^{249} \mathrm{Cf}$ and ${ }^{253} \mathrm{No}$ which are again based on the same configuration. However, the standard Woods-Saxon code, e.g., routinely predicts that the $\mathrm{j}_{15 / 2}$ neutron should align first. The recent work of Hota et al. 4 identifies that inclusion of higher order $\beta_{6}$ deformations shifts the predictions of neutron and proton alignment frequencies in the right direction and resolves earlier discrepancies in the predictions of the neutron and proton alignment frequencies. In this context, it would be instructive to bridge this study of $N=151$ isotones with ${ }^{251} \mathrm{Fm}$.

A strong fingerprint of the single-particle configuration in odd-mass nuclei can be provided by the signature splitting. In the actinide region, good examples are provided by the study of ${ }^{237} \mathrm{~Np}$ and ${ }^{241} \mathrm{Am}[2]$ where the signature splitting of the $\pi 5 / 2^{+}[642]$ and $\pi 5 / 2^{-}[523]$ orbitals are significant.

In the $K=1 / 2$ case, the decoupling of the bands has consequences not only on the energy levels, but also on the relative population intensity of the signature partners. If the decoupling is significant, it results in a dominance of $E 2$ transitions in the favoured partner. Typical examples are provided in ${ }^{249} \mathrm{Cm}\left(\nu 1 / 2^{+}[620]\right)$ [7] and ${ }^{251} \mathrm{Md},{ }^{255} \operatorname{Lr}\left[16,30\right.$ ] with the $\pi 1 / 2^{-}$[521] orbital from the $2 \mathrm{f}_{5 / 2}$ spherical shell. The decoupling of the signature partners is well reproduced by calculations, though with some differences in the splitting magnitude at low rotational frequencies: see [16, 99, 105, 108, 116].

The magnetic properties are probably the most sensitive to compare with model predictions and/or to make configuration assignments. Depending on the model, different formalisms are used but basically the magnetic moment $\mu$ or gyromagnetic factor $g_{K}$ are related to the reduced transition probability $\mathrm{B}(M 1)$, then to the radiative transition rate $\mathrm{T}_{\gamma}(M 1)$ using equations 4, 8 , The magnetic moment is a strong function of the orbital properties (spin projection along the symmetry axis, $K$, and whether it is a proton or a 
neutron) and therefore a relevant indicator of the single-particle configuration. Usually, the ratio $\mathrm{T}(\Delta I=1 \hbar, M 1+E 2) / \mathrm{T}(E 2)$ is extracted from the data and compared with theoretical predictions. It is immediately clear that sensitivity to $M 1$ transitions is needed, which requires substantial gammaray statistics and conversion-electron detection if possible. Such intensity ratio analyses have been performed in ${ }^{245} \mathrm{Pu},{ }^{249,249} \mathrm{Cf}$ [7, 4], ${ }^{253} \mathrm{No}$ [22], ${ }^{251} \mathrm{Md}$ [16], ${ }^{255} \operatorname{Lr}$ [30, 66]. Theoretical predictions are usually made using public codes based on Woods-Saxon potentials [118, 119], by experimentalists themselves [4, 7, 22]. Unfortunately very few self-consistent HFB calculations in odd-mass transfermium nuclei have been performed except by Chatillon et al. [16] using the formalism detailed by Hamamoto and Sagawa [117. The results of such calculations are shown in Fig. 18 [120] for the rotational bands in ${ }^{251} \mathrm{Md}$ based on the Nilsson proton orbitals $1 / 2^{-}[521]$, $7 / 2^{-}[514]$ and $7 / 2^{+}[633]$. The plots show that for the $1 / 2^{-}[521]$ configuration, the unfavoured signature partner band $\alpha=-1 / 2$ will predominantly deexcite towards the favoured signature via $M 1$ transitions. For the $7 / 2^{-}[514]$ $\left(7 / 2^{+}[633]\right)$ configuration, $M 1$ transitions are expected to dominate below $I \sim 10 \hbar(I \sim 16 \hbar)$.

\subsection{Discussion of two quasiparticle states}

The main observables related to the structure of two quasiparticle states are spin, parity and excitation energy, and in the case of isomeric states, the decay lifetime and deduced $K$ value. As in the case of odd-mass nuclei, the rotational band properties can also provide insight into the two quasiparticle configuration. Since the structure of the two quasiparticle isomeric states and of the rotational band built upon them are closely intertwined, the following discussion is related to these two aspects. Whilst a number of two quasiparticle isomeric states are known in the transuranium region, studies of the associated rotational bands are still the exception rather than the rule. Theoretical investigations reflect the experimental status: still very few theoretical works are devoted to rotational bands built on high- $K$ isomeric states. In the following the discussion focusses on the $Z \geq 100{ }^{250} \mathrm{Fm}$, ${ }^{252,254}$ No nuclei which all display a $K^{\pi}=8^{-}$isomeric state and for which both in-beam and decay spectroscopy has been performed (although not yet fully successful in ${ }^{254} \mathrm{No}$ ).

As discussed above, when the spin and parity of the isomeric state has been experimentally constrained, there are very few single-particle orbitals available to couple to $K^{\pi}$; the discussion is often restricted to whether the 

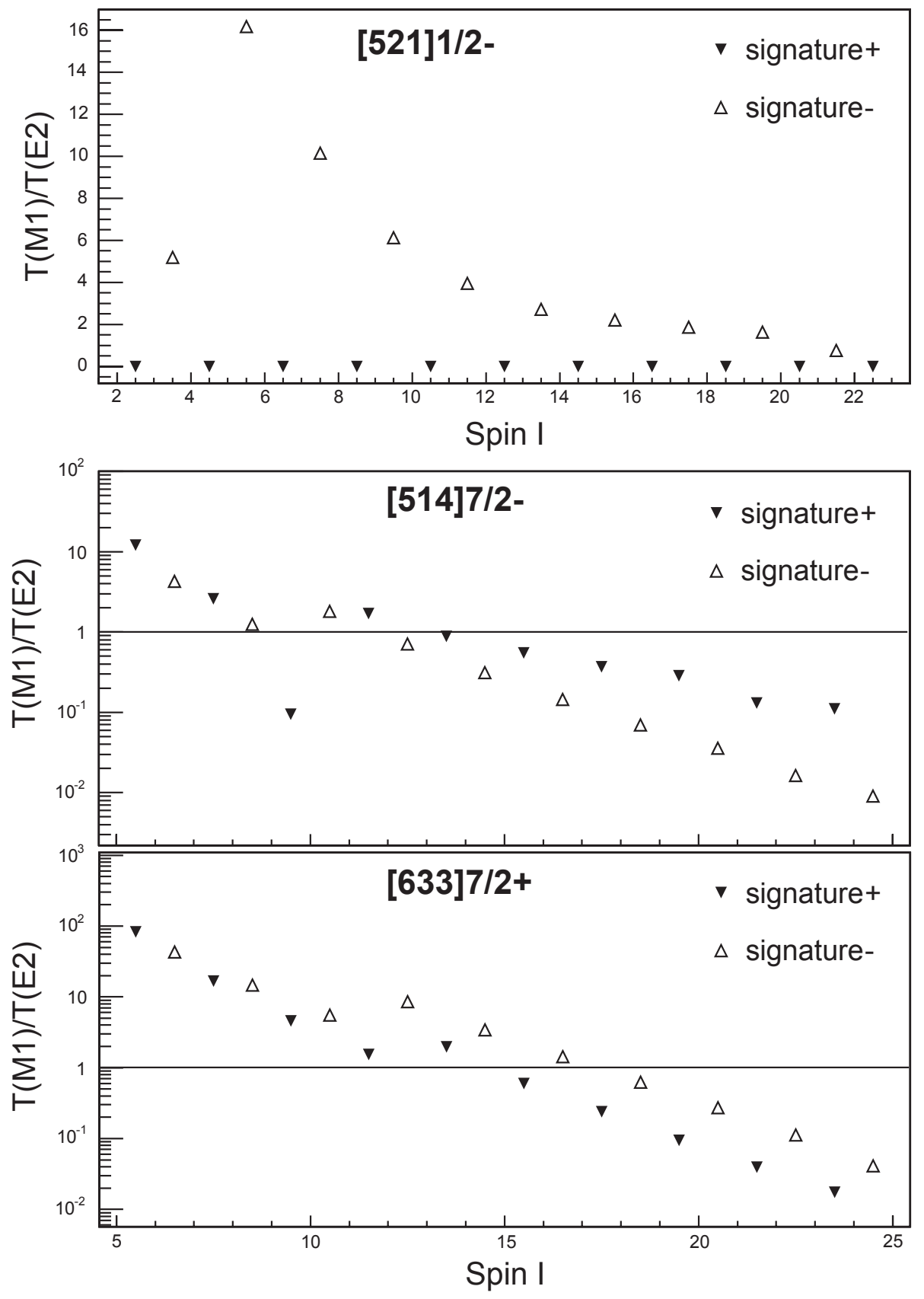

Figure 18: Ratio of the total (radiative and conversion electrons) transition rates $\mathrm{T}(M 1) / \mathrm{T}(E 2)$ for ${ }^{251} \mathrm{Md}$ [120] calculated using the HFB + Sly4 formalism for the rotational bands based on the Nilsson proton orbitals $1 / 2^{-}[521], 7 / 2^{-}[514]$ and $7 / 2^{+}[633]$. 
coupling concerns neutrons or protons. Obviously, the excitation energy and study of the isomeric decay path can also guide the distinction between proton and neutrons. In general, microscopic-macroscopic models reproduce rather well the single-particle sequence, especially the deformed shell gap at $Z=100, N=152$. These microscopic-macroscopic models can also reproduce the energies of high- $K$ isomeric states rather well, to within a few hundred keV. Due to the fact that in many treatments the underlying single-particle spectrum is not well reproduced, the disagreement for density functional based models can be as large as one $\mathrm{MeV}$. This general observation concerning the density-functional based models is discussed further below. The single-particle spectrum calculated (for ${ }^{250} \mathrm{Fm}$ ) with the Woods-Saxon potential is shown in Fig. 13 -a. A $K^{\pi}=8^{-}$two quasiparticle state can be built by coupling either the unpaired $7 / 2^{-}[514]$ and $9 / 2^{+}[624]$ protons or the $7 / 2^{+}[624]$ and $9 / 2^{-}[734]$ neutrons. In the $N=150$ isotopes, raising a neutron from the $7 / 2^{+}[624]$ orbital to the $9 / 2^{-}[734]$ orbital costs, besides pair breaking, little energy while in the $N=152$ isotones, breaking the $7 / 2^{+}[624]$ or $9 / 2^{-}[734]$ pairs involves excitation across the $N=152$ gap. On the proton side, the unpaired $7 / 2^{-}[514]$ and $9 / 2^{+}[624]$ configurations are more favoured in the $Z=102$ isotopes than for $Z=100$ where excitation across the deformed gap is needed. Using these simplified arguments, one therefore expects that the neutron $K^{\pi}=8^{-}$configuration is favoured in ${ }_{150}^{250} \mathrm{Fm}_{100}$, the proton $K^{\pi}=8^{-}$configuration in ${ }_{152}^{254} \mathrm{No}_{102}$ while neutron and proton $K^{\pi}=8^{-}$ configurations compete in ${ }_{150}^{252} \mathrm{No}_{102}$.

Table 3 shows predictions using microscopic-macroscopic models for these three isotopes. The states with $K_{+}=K_{1}+K_{2}$ and $K_{-}=\left|K_{1}-K_{2}\right|$ are degenerate in first order. It has long been known that the residual spin-spin interaction removes the degeneracy, lowering the spin singlet coupling and raising the triplet state (Gallagher rule [93]). In density functional theory calculations, time-reversal symmetry breaking seems to have the same effect as the spin-spin interaction [19, 106], although this has not been definitively established. Including the residual interaction or symmetry breaking lowers the singlet-coupling state by $\sim 100 \mathrm{keV}$ in the heaviest nuclei: see e.g. [81, 106, 124]. It should be noted that recoupling of the particles is not always included in the calculations (and is sometimes even not documented in the publications). To some extent this explains why calculations using the same model can lead to different predictions. It however remains surprising that calculations using a priori the same ingredients lead to a large spread in predictions and in particular inversions in the $K^{\pi}=8^{-}$proton and neutron 
energies for ${ }^{254}$ No. On the other hand, the situation is not as ambiguous in ${ }^{250} \mathrm{Fm}$ and ${ }^{252}$ No where all microscopic-macroscopic calculations favour a neutron $K^{\pi}=8^{-}$configuration.

For completeness, we mention that residual proton-neutron interactions of $\sim 200 \mathrm{keV}$ are well-established in the rare-earth region (see, for example, Ref. 94]). When proton and neutron 2-qp states lie within this range, this interaction will lead to mixing and energy shift, which should be included for accurate energy and configuration predictions.

The picture changes in a quite radical way when considering self-consistent calculations using the Skyrme or Gogny force. Figure 13-b displays a singleparticle spectrum for ${ }^{250} \mathrm{Fm}$ calculated using HFB + Gogny D1S force [70]. On the neutron side, there are two almost equally-sized deformed gaps at $N=150$ and 152. This has little consequence for the $N=150$ isotones whose $K^{\pi}=8^{-}$neutron states are predicted at $1029 \mathrm{keV}$ and $1031 \mathrm{keV}$ for ${ }^{250} \mathrm{Fm}$ and ${ }^{252}$ No respectively [19] (including the time-reversal symmetry breaking). Regarding the neutron $K^{\pi}=8^{-}$state, it is predicted at an excitation energy of over $2 \mathrm{MeV}$ in ${ }^{254} \mathrm{No}$. On the proton side, no proton deformed shell gap is predicted at $Z=100$ and the $9 / 2^{-}[734]$ orbital is predicted at too high excitation energy since the $\pi \mathrm{i}_{13 / 2}$ spherical orbital is also shifted to higher energy. As a consequence, the proton $\pi 7 / 2^{-}[514] \otimes \pi 9 / 2^{+}[624]$ configuration is predicted at too high energy in all isotopes [106. The situation is rather similar with HFB calculations using the Skyrme Sly4 force: see Fig. 13-c. Using this formalism, the $K^{\pi}=8^{-}$proton isomer is predicted at too high energy and a modest agreement is obtained for the $K^{\pi}=8^{-}$neutron states. Using the SkI4 force better reproduces the deformed $Z=100$ gap but at the price of a deterioration of the neutron single-particle spectrum [71].

While the excitation energy increases, the onset of other degrees of freedom is expected to occur. As discussed in e.g. [127], including particlevibration coupling improves the spectrum quality. Dynamic calculations have been performed in the transfermium region using either phonon operators [121, 128], Random Phase Approximation (RPA) [79, 124, 129], HFB configuration mixing [106]. The vast majority of these studies relate to low spin vibrations which have been observed experimentally in some cases in the $A \sim 250$ region. In particular $K^{\pi}=2^{-}$octupole vibrations have been observed at $\sim 900 \mathrm{keV}$ in ${ }^{250} \mathrm{Fm}\left[15\right.$ ] and ${ }^{252} \mathrm{No}$ [19, 79], and in other $N=150$ isotones. The only RPA calculation so far performed by Solov'ev et al. [129] in ${ }^{250} \mathrm{Fm}$ and ${ }^{254}$ No suggests that the $K^{\pi}=8^{-}$isomers are essentially pure two quasiparticle states, therefore without major vibrational component. 


\begin{tabular}{|c|c|c|c|c|c|}
\hline Config. & $\begin{array}{l}\mathrm{E}\left({ }^{250} \mathrm{Fm}\right) \\
(\mathrm{MeV})\end{array}$ & $\begin{array}{l}\mathrm{E}\left({ }^{252} \mathrm{No}\right) \\
(\mathrm{MeV})\end{array}$ & $\begin{array}{l}\mathrm{E}\left({ }^{254} \mathrm{No}\right) \\
(\mathrm{MeV})\end{array}$ & Reference & Model \\
\hline $\begin{array}{l}\nu \nu \\
\pi \pi\end{array}$ & & & $\begin{array}{l}1.2 \\
1.1\end{array}$ & $\begin{array}{l}\text { Ivanova } \\
\text { et al. [121] }\end{array}$ & $\begin{array}{l}\text { WS }+ \text { independent } \\
\text { qp model }\end{array}$ \\
\hline $\begin{array}{l}\nu \nu \\
\pi \pi\end{array}$ & 0.97 & & $\begin{array}{l}1.12 \\
1.48\end{array}$ & $\begin{array}{l}\mathrm{Xu} \text { et al. } \\
{[73]}\end{array}$ & PES WS+LN \\
\hline $\begin{array}{l}\nu \nu \\
\pi \pi\end{array}$ & & & $\begin{array}{l}1.27 \\
1.52\end{array}$ & $\begin{array}{l}\text { Herzberg } \\
\text { et al. } 84\end{array}$ & $\begin{array}{l}\text { Projected } \\
\text { Shell Model }\end{array}$ \\
\hline $\begin{array}{l}\nu \nu \\
\pi \pi\end{array}$ & & & $\begin{array}{l}1.8^{a} \\
1.296^{a}\end{array}$ & $\begin{array}{l}\text { Tandel } \\
\text { et al. } 85 \text {. }\end{array}$ & $\mathrm{WS}+\mathrm{LN}{ }^{b}$ \\
\hline $\begin{array}{l}\nu \nu \\
\pi \pi\end{array}$ & & $\begin{array}{l}\sim 1 . q^{a} \\
\sim 1.4^{\bar{a}}\end{array}$ & $\begin{array}{l}\sim 2.0^{\vec{a}} \\
\sim 1.4^{\bar{a}}\end{array}$ & $\begin{array}{l}\text { Kondev } \\
\text { et al. } 122 \text {. }\end{array}$ & $\mathrm{WS}+\mathrm{LN}$ \\
\hline $\begin{array}{l}\nu \nu \\
\pi \pi\end{array}$ & $\begin{array}{l}\sim 0.9 \\
\sim 2.2\end{array}$ & $\begin{array}{l}\sim 0.9 \\
\sim 1.25\end{array}$ & $\begin{array}{l}\sim 1.7 \\
\sim 1.3\end{array}$ & $\begin{array}{l}\text { Greenlees } \\
\text { et al. } 15\end{array}$ & $\begin{array}{l}\text { WS + quenched } \\
\text { pairing }\end{array}$ \\
\hline $\begin{array}{l}\nu \nu \\
\pi \pi\end{array}$ & $\sim 1.0^{a}$ & $\sim 1.0^{a}$ & $\begin{array}{l}1.1^{a} \\
2.0^{a}\end{array}$ & $\begin{array}{l}\text { Robinson } \\
\text { et al. } 79 \text { ] }\end{array}$ & $\mathrm{WS}+\mathrm{LN}$ \\
\hline $\begin{array}{l}\nu \nu \\
\pi \pi\end{array}$ & $\begin{array}{l}\sim 1.14 \\
\sim 1.50\end{array}$ & $\begin{array}{l}\sim 1.20 \\
\sim 1.36\end{array}$ & $\begin{array}{l}\sim 1.46 \\
\sim 1.28\end{array}$ & $\begin{array}{l}\text { Adamian } \\
\text { et al. [123. }\end{array}$ & $\begin{array}{l}\text { Two Center } \\
\text { Shell Model }\end{array}$ \\
\hline $\begin{array}{l}\nu \nu \\
\pi \pi\end{array}$ & $1.174^{a}$ & $\begin{array}{l}1.244^{a} \\
1.336\end{array}$ & $\begin{array}{l}1.249^{a} \\
1.400^{a}\end{array}$ & $\begin{array}{l}\text { Jolos et al. } \\
{[124]}\end{array}$ & $\begin{array}{l}\text { Quasi-particle } \\
\text { phonon model }{ }^{c}\end{array}$ \\
\hline $\begin{array}{l}\nu \nu \\
\pi \pi\end{array}$ & $\begin{array}{l}\sim 1 \\
\sim 2.2\end{array}$ & & $\begin{array}{l}1.357 \\
1.378\end{array}$ & $\begin{array}{l}\text { Liu et al. } \\
{[125,126]}\end{array}$ & $\mathrm{PES} \mathrm{WS}+\mathrm{LN}$ \\
\hline
\end{tabular}

Table 3: Energy predictions of $K^{\pi}=8^{-}$states in ${ }^{250} \mathrm{Fm},{ }^{252} \mathrm{No}$ and ${ }^{254} \mathrm{No}$ using microscopic-macroscopic models. The following abbreviations are used: WS $=$ WoodsSaxon; PES = Potential Energy Surface; LN = Lipkin-Nogami.

${ }^{a}$ Degeneracy removal by inclusion of the residual spin-spin interaction or of timereversal partners explicitly indicated.

${ }^{b}$ Pairing strength readjusted to reproduce mass differences in the ${ }^{254}$ No region.

${ }^{c}$ WS-based, no vibration included for these two quasiparticle states 
Calculations discussed until now in this section assume reflection symmetric shapes. Static octupole $Y_{32}$ correlations breaking this symmetry have been suggested in the transfermium region using multidimensional constrained covariant density functional theory [130] or the reflection asymmetric shell model [131]. In this last reference, the excitation energy of the $2^{-}$reflection asymmetric state and of the rotational band built on it are remarkably well reproduced in ${ }^{246} \mathrm{Cm},{ }^{248} \mathrm{Cf},{ }^{250} \mathrm{Fm}$ and ${ }^{252} \mathrm{No}$. By extension, the $K^{\pi}=8^{-}$ isomers could also exhibit an octupole component. On the other side, HFB calculations using the D1S Gogny force do not predict static $\beta_{3}$ deformation until $N \sim 186$ [132].

Calculations of rotational bands built on high- $K$ isomeric states are still seldomly performed in the $A \sim 250$ mass region. So far such calculations have been reported only by Sulignano et al. [19] and Fu et al. [133. in both ${ }^{250} \mathrm{Fm}$ and ${ }^{252}$ No. Sulignano et al. performed cranked HFB calculations using the D1S Gogny force. Remarkable agreement with the experimental $\mathcal{J}^{(1)}$ and $\mathcal{J}^{(2)}$ moment of inertia was obtained. Fu et al. [133] have performed Configuration Constrained Total Routhian Surface (CCTRS) calculations, which again give good reproduction of the moments of inertia. The latter work was triggered by the fact the dynamic moment of inertia $\mathcal{J}^{(2)}$ in both ${ }^{250} \mathrm{Fm}$ and ${ }^{252}$ No displays an irregularity at $\hbar \omega \sim 0.2 \mathrm{MeV}$, as shown in Fig. 16 . Such irregularities are usually due to the crossing of two rotational bands. It was suggested in [19] that the two quasiparticle band based on the $K^{\pi}=8^{-}$ isomer could cross the rotational band based on the $K^{\pi}=2^{-}$excitation, but a crossing with a yet unobserved $K^{\pi}=7^{-}$neutron band was not excluded. In their calculations, $\mathrm{Fu}$ et al. nicely reproduce the irregularity in the moment of inertia, indeed associated with a band crossing but with however a $K^{\pi}=7^{-}$proton configuration. It is clear, however, that reproducing a band crossing requires that the two bands must have the correct relative energy, which is well beyond the level of accuracy of the models. It is, however, equally true that the experimental finding of [19] and theoretical calculations of [133. converge on the underlying mechanism leading to the irregularity in the moment of inertia. Note that the theoretical analysis based on HFB with D1S Gogny force [19] did not display any neutron orbital crossing.

The single particle assignment of $K^{\pi}=8^{-}$isomeric states in ${ }^{250} \mathrm{Fm}$ and ${ }^{252} \mathrm{No}$ is confirmed by the electromagnetic properties of the band, namely the $T(\Delta I=1 \hbar) / T(E 2)$ ratio linked to the magnetic moment (or gyromagnetic factor). Unfortunately, the two cranking approaches of Sulignano et al. [19] and $\mathrm{Fu}$ et al. [133] discussed above do not provide such quantities for 
comparison. In fact, Greenlees et al. [15] and Sulignano et al. [19] compare the experimental intensity ratio using gyromagnetic factors calculated with the Woods-Saxon potential [119]. It is worth noting that the controversial single-particle assignment of the $K^{\pi}=8^{-}$isomer in ${ }^{254}$ No could be resolved as soon as a rotational band built on it will be observed. It is not clear why such a band has not yet been observed using prompt spectroscopy while they have been found in ${ }^{250} \mathrm{Fm}$ and ${ }^{252} \mathrm{No}$ populated with a lower cross-section.

\subsection{General remarks}

As discussed above, it is now becoming apparent that self-consistent mean-field calculations seem to suffer from the same symptom: the deformed shell gaps at $Z=100$ and $N=152$ are not well reproduced (see e.g. [104, 110, 71, 127, 134]). This has not only consequences on the predicted properties of deformed nuclei but also on the predictions for spherical nuclei. These deficiencies in the description of the single-particle spectrum do not greatly affect the predictions of rotational properties such as the moments of inertia, but are of importance when attempting to reproduce the excitation energies of one and two quasiparticle states. Attempts to address these deficiencies are underway. In the work of Shi et al., the spin-orbit coupling constants of UNEDF1, a recent Skyrme parametrisation [135], and the pairing strengths were adjusted in order to better reproduce the excitation spectra and oddeven mass differences [110]. This local adjustment resulted in the opening of the deformed shell gaps at $Z=100$ and $N=152$ and also of the $Z=114$ spherical shell gap at sphericity. See also the discussion in [71].

\section{Prospects and Future Perspectives}

As has been demonstrated, in-beam gamma-ray studies of even-even (super)heavy nuclei are now feasible down to a production cross-section at the level of $10 \mathrm{nb}$. However, relatively few in-beam studies of nuclei beyond plutonium have been performed, as evidenced by table 1. As far as odd-mass nuclei are concerned, it is a striking fact that high-spin states are known in only a very few cases. This is understandable for transfermium isotopes where the production cross-sections for fusion-evaporation reactions are in the best cases at the $\mu \mathrm{b}$ level. The limiting case in studies of odd-mass nuclei is ${ }^{255} \mathrm{Lr}$, successfully studied in-beam at the level of $200 \mathrm{nb}$ [62]. The lack of high-spin data is even more surprising in the $Z=93-99$ region where, without exception, 
such states have been studied using either inelastic scattering or transfer reactions involving only the transfer of a few nucleons. Going beyond the present studies in this region can be achieved either using a larger variety of actinide targets or using fusion-evaporation reactions, but in both cases severe limitations are imposed. The availability of actinide targets is very limited not only because of their lifetime, but also because the activity of the target material causes a high gamma-ray counting rate in the absence of beam and makes the in-beam measurement much more challenging. When considering fusionevaporation reactions, there are broadly two classes - "cold" fusion reactions, where the compound nucleus excitation energy is low with only one to three neutrons emitted, or more asymmetric "hot" fusion reactions where typically four or more neutrons are emitted due to the high compound nucleus excitation energy. Cold fusion reactions have been used to good effect and there are still quite a number of nuclei accessible using beams such as ${ }^{48} \mathrm{Ca}$ or ${ }^{36} \mathrm{~S}$. To give an example from the einsteinium isotopes, there appears to be no literature concerning the use cold fusion-evaporation reactions to produce these nuclei. From a recent study at JYFL, a cross-section of less than $50 \mathrm{nb}$ has been measured for the reaction ${ }^{197} \mathrm{Au}\left({ }^{48} \mathrm{Ca}, 2 n\right){ }^{243} \mathrm{Es}$ [65], which is probably beyond the current limits for an odd-mass nucleus in an in-beam gamma-ray spectroscopic study. As these reactions are not too asymmetric, the transmission efficiency through recoil separators is generally good (at the level of 35-50\%). The alternative approach is to use "hot" fusion-evaporation reaction such as ${ }^{238} \mathrm{U}\left({ }^{14} \mathrm{~N}, x n\right){ }^{252-x}$ Es which are at the $\mu$ b level [136]. In such cases, use of the recoil-decay tagging technique is more challenging due to the reduced transmission through the recoil separator devices. In vacuum mode devices, a velocity filter can be used but the separator transmission is usually low because of the large recoil angular and momentum dispersions. The use of a gas-filled separator is also challenging for these very asymmetric reactions, as in addition to the angular dispersion, the recoiling fusion-evaporation products have very low velocity and are subject to additional energy loss in the filled gas. To date, the only successful in-beam recoil-decay tagging studies of heavy nuclei using asymmetric reactions have been performed at JYFL using RITU. These are the study of ${ }^{226} \mathrm{U}$ produced using the ${ }^{208} \mathrm{~Pb}\left({ }^{22} \mathrm{Ne}, 4 n\right){ }^{226} \mathrm{U}$ reaction, with a production cross section of approximately $10 \mu \mathrm{b}$ and a transmission through the recoil separator of around $10 \%$ [137], and the more recent study of ${ }^{222} \mathrm{Th}$ using the ${ }^{208} \mathrm{~Pb}\left({ }^{18} \mathrm{O}, 4 n\right){ }^{222} \mathrm{Th}$ reaction, with a production cross section of approximately $4 \mathrm{mb}$ and slightly lower transmission. The latter experiment employed the SAGE spectrome- 
ter for combined in-beam gamma-ray and conversion electron spectroscopy. Example spectra from this study can be found in ref. [43]. As yet, no asymmetric hot fusion-evaporation reaction using actinide targets have successfully been used for in-beam spectroscopic studies. This is mainly due to the fact that the required combination of efficient germanium detectors array, high-acceptance recoil separator and possibility to use actinide targets has not been realised. As an example, the "doubly-magic" deformed ${ }^{252} \mathrm{Fm}$ with both proton number and neutron number corresponding to a deformed shell gap could be studied using the reaction ${ }^{238} \mathrm{U}\left({ }^{18} \mathrm{O}, 4 n\right){ }^{252} \mathrm{Fm}$ with a cross section of $\sim 750 \mathrm{nb}[138$.

Several studies suggested the possibility of using symmetric reactions (with a view to using neutron-rich Xe or Sn beams in a future radioactive beam facility) see e.g. [139, 140, 141, 142, 143, 144, 145, 146]. The predicted cross sections are in some cases controversial and can differ by several orders of magnitude. The experimental investigations which have been made to date are moreover not encouraging: see [147, 148]. Such symmetric reactions are also not suited to many recoil separator devices, as the velocities of the beam and reaction products become much closer. However, should it be possible to separate the products, the transmission will be very high due to the low angular spread of the recoil cone.

Multinucleon transfer reactions using actinide targets have been used to produce heavy elements on the neutron rich side of the nuclear chart up to ${ }^{256} \mathrm{Es},{ }^{257} \mathrm{Fm},{ }^{260} \mathrm{Md},{ }^{259} \mathrm{No},{ }^{262} \mathrm{Lr}$ : see the contribution by Kratz, Loveland and Moody in this volume and e.g [149] and references therein. In these studies, nuclei were identified after chemical separation techniques; cross-sections up to several mb were measured in some cases [150]. There is renewed interest from both the experimental and theoretical point of view in using such reactions, given their impressive potential for synthesis and spectroscopy, as can be seen in Refs. [151, 152, 153] and references therein. Experimentally, the challenge is to identify the mass and charge of the nuclei of interest produced in a large angular and momentum range, keeping in mind that they are not necessarily alpha emitters and that a rather large number of different reaction products can be accessed: see e.g. the recent study using AGATA, the PRISMA spectrometer and the reaction ${ }^{136} \mathrm{Xe}+{ }^{238} \mathrm{U}$ [154]. Undoubtedly, further studies and/or experimental developments are needed before in-beam spectroscopy of multinucleon transfer products can be made in this mass region.

Much the same discussion regarding reactions can be applied to the search 
for multi-quasiparticle states, though in that case, the experimenter must often be guided by theoretical predictions of nuclei where high- $K$ states are likely to be found. In some cases, the interpretation of experimental data is still open with ${ }^{254}$ No being a good example. The decay study of ${ }^{250} \mathrm{No}$ by Peterson et al. [83] shows evidence for an isomeric state whose lifetime is longer than the ground-state, but the level scheme is not well established and the experiment could not distinguish between direct fission or decay via an electromagnetic branch. Clearly this is another case which deserves further investigation.

The two quasiparticle states in even-even nuclei have their counterpart in odd-mass nuclei where three quasiparticle states can be found. Such three quasiparticle isomeric states have been reported in the transfermium nuclei ${ }^{253}$ No [155, ${ }^{255} \operatorname{Lr}$ [67, 66] and ${ }^{257} \operatorname{Rf}$ [57, 156, 157]. However, the study of such states is clearly very challenging and the corresponding level schemes are still somewhat tentative. That said, the level schemes already provide evidence for rotational bands which are fed by the decay of the isomeric state.

Finally, evidence for a four quasiparticle isomeric state has been presented in ${ }^{254} \mathrm{No}\left(t_{1 / 2} \sim 180 \mu \mathrm{s}, \mathrm{E}=2.5-3 \mathrm{MeV}\right)$ [84, 85, 86, 87] and ${ }^{256} \mathrm{Rf}$ [88, 91, 89], the excitation energy and structure of which is still controversial. Theoretical calculations of four quasiparticle configurations are still scarce: see Liu et al. [126] for Fm to Cm isotopes, and Kondev et al. [122] for No and Rf isotopes. Detailed studies would certainly offer more insight into the singleparticle structure, and in-beam gamma-ray spectroscopy of rotational bands based on such multi-quasiparticle states is certainly a challenge for the future.

As stated, so far the number of nuclei studied in the $A \sim 250$ mass region is rather low. The "doubly-magic" deformed ${ }^{252} \mathrm{Fm}$ with both proton and neutron number coinciding with a deformed shell gap has not yet been studied using in-beam spectroscopy. A push should be made in the direction of the next deformed shell gaps at $Z=108, N=162$ possibly reaching the corresponding isotope ${ }^{270} \mathrm{Hs}$. In addition, a push toward the $\beta$-line of stability to the more neutron-rich side of the nuclei studied to date would also be of interest. The multi-nucleon transfer reactions described above are of interest in this respect. The lighter actinide nuclei should also not be neglected, as potentially higher cross sections could allow more precise measurements to be made. Using radioactive neutron-rich beams is an alternative: see e.g. [158, 159]. Although exciting due to the possibility of producing new isotopes, intensities which will be unattainable for at least the short or mid-term would be required to perform in-beam spectroscopy. The 
dumping of the radioactive beam and possible build-up of radioactivity in the target chamber are additional challenging issues which must be overcome.

Large efforts are and should be devoted to providing more efficient arrays of germanium detectors and to improve the recoil separator and spectrometer devices. The Advanced GAmma-ray Tracking Array AGATA [160] and GRETINA [161, the first stage of GRETA (Gamma-Ray Energy Tracking Array), are the latest generation of germanium detector arrays based on the concept of photon tracking in a shell of highly-segmented germanium crystals. Efficiencies as large as $30 \%$ at $1 \mathrm{MeV}$ are expected for a $3 \pi$ array, therefore a gain of a factor of $\sim 3$ compared to arrays based on Comptonsuppressed germanium detectors whose maximum efficiencies culminate at $\sim 10 \%$. An even more impressive gain factor should be expected for high-fold coincidence measurements that are essential for the study of odd-mass nuclei where the decay path is much more fragmented. New algorithms to allow higher data rates and higher beam intensities without losses in throughput or spectrum quality should also be developed.

In parallel, new high acceptance gas-filled separators are being constructed. At Argonne, a gas-filled analyser (AGFA) is being constructed for experiments with the ATLAS accelerator. AGFA consists of a large-bore quadrupole and a dipole magnet, shaped to give higher-order multipole components for focusing. For evaporation residues in the ${ }^{208} \mathrm{~Pb}\left({ }^{48} \mathrm{Ca}, 2 \mathrm{n}\right){ }^{254}$ No reaction, Monte Carlo trajectory simulations indicate a transmission efficiency of $70 \%$ to a $64 \times 64 \mathrm{~mm}$ detector [162]. The two distinct features of AGFA are (i) large efficiency can be achieved while accommodating 100 detectors of GAMMASPHERE, with an full-energy peak efficiency of $9 \%$ at $1.3 \mathrm{MeV}$, and (ii) evaporation residues are focused into a smaller area, which leads to larger detection efficiency of Ge detectors surrounding the focal-plane detector used for decay spectroscopy. GAMMASPHERE has been upgraded from analogue to digital electronics to accommodate higher counting rates. Compared to the current ATLAS setup of the FMA and GAMMASPHERE, the new setup should give a 50-fold increase in data accumulation rate for in-beam spectroscopy, including a unique calorimetric capability for fission barrier measurements. Compared to JUROGAM II and RITU at JYFL, the gain factor will be approximately 5-fold. For decay spectroscopy, e.g. of isomers, at the focal plane, the estimated improvement is by a factor of approximately 100. The latter figure takes into account that ATLAS has been upgraded to accelerate highintensity beams of over $1 \mu \mathrm{A}$, providing a competitive new facility for such 
studies.

After successful tests of the vamos spectrometer $\left(\mathrm{Q}_{v} \mathrm{Q}_{h} \mathrm{D}\right.$ configuration i.e. vertical- followed by horizontal-focussing electric quadrupole, then magnetic dipole) in gas-filled mode were performed [163], an upgrade is being implemented for campaigns with AGATA, EXOGAM, PARIS [164] and the MUSETT focal plane array 165 . A transmission $\times$ detection efficiency of $95 \%$ has been measured using the ${ }^{40} \mathrm{Ca}+{ }^{150} \mathrm{Sm}$ reaction. The combination of tracking arrays and large acceptance separators should allow the spectroscopic limit to drop below the $1 \mathrm{nb}$ barrier and give access to isotopes up to $\mathrm{Bh}$.

In developing new recoil separator devices, attention should also be paid to the suppression of unwanted products reaching the focal plane. As the cross-section for fusion-evaporation goes down, the rate of background events entering the selection "gates" for fusion products becomes similar if not higher than that of the nucleus of interest. In turn, this means that a selection of gamma rays of interest on the basis of recoil gating alone is not sufficient to obtain a clean gamma-ray spectrum. Such effects were already apparent in the studies of ${ }^{246} \mathrm{Fm}$ and ${ }^{256} \mathrm{Rf}$ at RITU, where alpha and fission tagging were required, respectively. As noted previously, in-beam spectroscopy using very asymmetric hot fusion reactions has only been performed in isotopes of thorium and uranium. The development of separators better suited to such reactions would be valuable for accessing nuclei on the neutron-rich side of the region studied to date.

It should also be mentioned that in New Delhi, India, the HYRA, the HYbrid Recoil mass Analyser operated in a gas-filled mode has been coupled to a calorimeter (known as $4 \pi$ spin spectrometer) for reaction mechanism studies of heavy elements [166, 167]: see e.g. [168]. There are plans couple to this separator with the Indian National Gamma Array INGA [169].

It is, of course, interesting to explore the possibilities of using other techniques to study heavy elements. Large-area segmented planar germanium detectors under development promise an intriguing alternative for optimising the detection of 100-500 keV gamma-rays and characteristic x-rays in heavy and superheavy prompt spectroscopy [170, 171]. A thin detector would win over current large-volume co-axial detectors in improving signal-to-noise in the "hostile" high-radiation environment of superheavy experiments, as the high-energy gamma-rays would preferentially pass through without interaction. A compact box geometry of such detectors could subtend a large solid-angle at the target position, and the high degree of segmentation and 
short charge drift distances would allow a single crystal to count at rates approaching $1 \mathrm{MHz}$. The tolerances of such detectors to neutron damage and subsequent robustness to annealing procedures are currently under study and show excellent promise [170, 171]. For completeness, mention should also be made of the x-ray fluorescence technique which gives insight into the fission times of compound nuclei [172, recently applied to atomic number $Z=120$ [173]. More spectroscopic data can be gained measuring the lifetime of collective states using a plunger technique, though suitable reactions may be difficult to find. More sensitivity (related to the difference in energy between the partially and fully shifted components) would be obtained in inverse kinematics, which represents a challenge in terms of beam rejection of the separator/spectrometer. Coulomb excitation measurements can give access to the transition rates. Using inverse kinematics would provide enough energy to excite ${ }^{254}$ No with sufficient cross-section, providing that a suitable detection system is constructed around a secondary target. This would ideally combine conversion electron and gamma-ray detection, recoildecay tagging and measurement of the deflection angle of the scattered ions, which is not a straightforward task.

\section{Summary}

In this review, a brief overview of the major developments and results over the past five years or so from in-beam spectroscopic studies of heavy elements has been presented. The experimental information available on these heavy nuclei has been expanded in several directions, in terms of the number of nuclei for which in-beam data exists, as well as in terms of spin, excitation energy and the level of spectroscopic detail to which individual nuclei have been investigated. In many cases this detailed data has put experimental assignments on a much firmer footing, and has given new insights into phenomena such as single-particle energies, rotational alignment, shell and pairing effects. This experimental data has been used to confront theory, with interesting results. Indeed, the data can now be used to benchmark theoretical predictions, as demonstrated elsewhere in this volume. With the next generation of gamma-ray tracking arrays on the horizon, and the possibility to exploit and develop complementary devices, there is every reason to believe that progress in this field will continue. In the future, ever lower limits of sensitivity should be reached, allowing the push to higher $Z$ to continue and allowing even more detailed studies of nuclei at lower $Z$. This continued 
progress and ongoing dialogue with theory should enable our understanding of the structure of heavy elements to improve still further.

Some of this material is based upon work supported by the U.S. Department of Energy, Office of Science, Office of Nuclear Physics, under award numbers DE-FG02-94ER40848 and contract number DE-AC02-06CH11357. Some of this research used resources of ANL's ATLAS facility, which is a DOE Office of Science user facility. We are grateful to R. Briselet for providing new data prior to publication.

[1] R.-D. Herzberg and P.T. Greenlees, Prog. Part. Nucl. Phys. 61 (2008) 674.

[2] K. Abu Saleem et al., Phys. Rev. C 70 (2004) 024310.

[3] K. Abu Saleem, PhD Thesis, Illinois Institute of Technology (2002).

[4] S.S. Hota et al., Phys. Lett. B 739 (2014) 13.

[5] G. Hackman et al., Phys. Rev. C 57 (1998) R1056.

[6] T. Ishii et al., Phys. Rev. C 78 (2008) 054309.

[7] S.K. Tandel et al., Phys. Rev. C 82 (2010) 041301(R).

[8] T. Ishii et al., Phys. Atom. Nucl. 70 (2007) 1457.

[9] S.S. Hota, PhD Thesis, University of Massachusetts Lowell, 2012.

[10] R. Takahashi et al., Phys. Rev. C 81 (2010) 057303.

[11] Y. Qiu et al., to be published.

[12] J. Piot et al., Phys. Rev. C 85 (2012) 041301(R).

[13] S. Ketelhut, PhD Thesis, University of Jyväskylä, 2010.

[14] J.E. Bastin et al., Phys. Rev. C 73 (2006) 024308.

[15] P.T. Greenlees et al., Phys. Rev. C 78 (2008) 021303(R).

[16] A. Chatillon et al., Phys. Rev. Lett. 98 (2007) 132503. 
[17] R.-D. Herzberg et al., Phys. Rev. C 65 (2001) 014303.

[18] A.-P. Leppänen et al., Eur. Phys. J. A 28 (2006) 301.

[19] B. Sulignano et al., Phys. Rev. C 86 (2012) 044318.

[20] R.-D. Herzberg et al., Eur. Phys. J. A 15 (2002) 205.

[21] P. Reiter et al., Phys. Rev. Lett. 95 (2005) 032501.

[22] R.-D. Herzberg et al., Eur. Phys. J. A 42 (2009) 333.

[23] P. Reiter et al., Phys. Rev. Lett. 82 (1999) 509.

[24] M. Leino et al., Eur. Phys. J. A 6 (1999) 63.

[25] P. Reiter et al., Phys. Rev. Lett. 84 (2000) 3542.

[26] P.A. Butler et al., Phys. Rev. Lett. 89 (2002) 202501.

[27] R.D. Humphreys et al., Phys. Rev. C 69 (2004) 064324.

[28] S. Eeckhaudt et al., Eur. Phys. J. A 25, s01 (2005) 605 and Eur. Phys. J. A 26 (2005) 227.

[29] G. Henning et al., Phys. Rev. Lett. 113 (2014) 262505.

[30] S. Ketelhut et al., Phys. Rev. Lett. 102 (2009) 212501.

[31] P.T. Greenlees et al., Phys. Rev. Lett. 109 (2012) 012501.

[32] K.-H. Schmidt et al., Phys. Lett. B 168 (1986) 39.

[33] E.S. Paul et al., Phys. Rev. C 51 (1995) 78.

[34] C.N. Davids et al., Nucl. Instr. Meth. B 70 (1992) 358.

[35] M. Leino et al., Nucl. Instr. Meth. B 99 (1995) 653.

[36] I.H. Lazarus et al., IEEE Trans. Nucl. Sci. 48 (2001) 567.

[37] C.W. Beausang et al., Nucl. Instr. Meth. A 313 (1992) 37.

[38] L. Arnold et al., IEEE Trans. Nucl. Sci. 53 (2006) 723 . 
[39] P.J. Nolan, F.A. Beck, D.B. Fossan, Ann. Rev. Nucl. Part. Sci. 44 (1994) 561.

[40] J. Rubert et al., Nucl. Instr. Meth. B 276 (2012) 33.

[41] VHS-ADC from Nutaq (formerly Lyrtech): http://nutaq.com/en/ products/vhs-adc/.

[42] H. Kankaanpää et al., Nucl. Instr. Meth. A 534 (2004) 503.

[43] J. Pakarinen et al., Eur. Phys. J. A 50 (2014) 53.

[44] S.W. Yates, A.M. Friedman and I. Ahmad, Phys. Rev. C 12 (1975) 795.

[45] I. Ahmad, R.R. Chasman and A.M. Friedman, Phys. Lett. B 251 (1990) 338.

[46] R.R. Chasman, I. Ahmad, A.M. Friedman and J.R. Erskine, Rev. Mod. Phys. 49 (1977) 833.

[47] M. Bender, W. Nazarewicz and P.-G. Reinhard, Phys. Lett. B 515 (2001) 42.

[48] A.Sobiczewski and K. Pomorski, Prog. Part. Nucl. Phys. 58 (2007) 292.

[49] T. Ishii et al., J. Phys. Soc. Japan 75 (2006) 043201.

[50] B.B. Back, H.C. Britt, O. Hansen, B. Leroux, and J.D. Garrett, Phys. Rev. C 10 (1974) 1948 .

[51] Ronald E. Brown, J.A. Cizewski, E.R. Flynn, and J.W. Sunier, Phys. Rev. C 20 (1979) 1301.

[52] H. Makii et al., Phys. Rev. C 76 (2007) 061301(R).

[53] M. Asai, Private Communication.

[54] A. Sobiczewski, I. Muntian, and Z. Patyk, Phys. Rev. C 63 (2001) 034306.

[55] J. Tuli, ENSDF data base, http://www.nndc.bnl.gov/ensdf/

[56] S. Harris, Phys. Rev. 138 (1965) B509. 
[57] J. Qian et al., Phys. Rev. C 79 (2009) 064319.

[58] G. Audi, A.H. Wapstra and C. Thibault, Nucl. Phys. A 729 (2003) 337.

[59] I. Wiedenhöver et al., Phys. Rev. Lett. 83 (1999) 2143.

[60] F.P. Heßberger et al., Z. Phys. A 359 (1997) 415.

[61] S. Eeckhaudt, PhD Thesis, University of Jyväskylä, 2006.

[62] F. Heßberger et al., Eur. Phys. J. A26 (2005) 233.

[63] A. Chatillon et al., Eur. Phys. J. A 30 (2006) 397.

[64] F. Déchery, PhD Thesis, Université Paris VII, 2012.

[65] R. Briselet, Private communication.

[66] H.B. Jeppesen et al., Phys. Rev. C 80 (2009) 034324.

[67] K. Hauschild et al., Phys. Rev. C 78 (2008) 021302(R).

[68] S. Antalic et al., Eur. Phys. J. A 38 (2008) 219.

[69] F.G. Kondev, G.D. Dracoulis and T. Kibédi, At. Data Nuc. Data Tab. 103-104 (2015) 50.

[70] J.-P. Delaroche, Priv. Comm.

[71] M. Bender and P.-H. Heenen, J. Phys.: Conf. Series 420 (2013) 012002.

[72] K.E.G. Löbner, Phys. Lett. B 26 (1968) 369.

[73] F.R. Xu, E.G. Zhao, R. Wyss and P.M. Walker, Phys. Rev. Lett. 92 (2004) 252501.

[74] A. Ghiorso, K. Eskola, P. Eskola and M. Nurmia, Phys. Rev. C 7 (1973) 2032.

[75] R.-D. Herzberg and D.M. Cox, Radiochim. Acta 99 (2011) 441.

[76] P. Chowdhury et al., AIP Conf. Proc. 1377 (2011) 13.

[77] Y.A. Akovali, Nuclear Data Sheets 99 (2003) 197. 
[78] U. Shirwadkar, PhD Thesis, University of Massachusetts Lowell, 2009.

[79] A.P. Robinson et al., Phys. Rev. C 78 (2008) 034308.

[80] S.K. Tandel. Proc. DAE Symp. on Nucl. Phys. 56 (2011) 99.

[81] K. Katori, I. Ahmad, A.M. Friedman, Phys. Rev. C 78 (2008) 014301.

[82] H.L. Hall et al., Phys. Rev. C 39 (1989) 1866.

[83] D. Peterson et al., Phys. Rev. C 74 (2006) 014316.

[84] R.-D. Herzberg et al., Nature 442 (2006) 996.

[85] S.K. Tandel et al., Phys. Rev. Lett. 97 (2006) 082502.

[86] F.P. Heßberger et al., Eur. Phys. J. A 43 (2010) 55.

[87] R.M. Clark et al., Phys. Lett. B 690 (2010) 19.

[88] H.B. Jeppesen et al., Phys. Rev. C 79 (2009) 031303(R).

[89] J. Rubert, P.T. Greenlees et al., to be published.

[90] S. Hofmann et al., Eur. Phys. J. A 10 (2001) 5.

[91] A.P. Robinson et al., Phys. Rev. C 83 (2011) 064311.

[92] G.D. Jones, Nucl. Instr. Meth. A 488 (2002) 471.

[93] C.J. Gallagher, Phys. Rev. 126 (1962) 1525.

[94] T.L. Khoo, J.C. Waddington, R.A. O'Neil, Z. Preibisz, D.G. Burke and M.W. Johns, Phys. Rev. Lett. 28 (1972) 1717.

[95] E. Parr, R.-D. Herzberg, S. Antalic, P.T. Greenlees, F.P. Heßberger, S. Ketelhut, and D. Rostron, Eur. Phys. J. A 48 (2012) 134.

[96] I. Ahmad, A.M. Friedman, R.R. Chasman and S.W. Yates, Phys. Rev. Lett. 39 (1977) 12.

[97] I. Ahmad, R.K. Sjoblom, A.M. Friedman and S.W. Yates, Phys. Rev. C 17 (1978) 2163. 
[98] Xiao-tao He, Zhong-zhou Ren, Shu-xin Liu and En-guang Zhao, Nucl. Phys. A 817 (2009) 45.

[99] Zhen-Hua Zhang, Xiao-Tao He, Jin-Yan Zeng, En-Guang Zhao and Shan-Gui Zhou, Phys. Rev. C 85 (2012) 014324.

[100] J.E. Draper et al., Phys. Rev. C 42 (1990) R1791.

[101] J. Becker et al., Phys. Rev. C 46 (1992) 889.

[102] C. Baktash, W. Nazarewicz, R. Wyss. Nucl. Phys. A 555 (1993) 375.

[103] R. Wyss and S. Pilotte, Phys. Rev. C 44 (1991) R602.

[104] A.V. Afanasjev et al., Phys. Rev. C 67 (2003) 024309.

[105] A.V. Afanasjev and O. Abdurazakov, Phys. Rev. C 88 (2013) 014320.

[106] J.-P. Delaroche, M. Girod, H. Goutte and J. Libert, Nucl. Phys. A 771 (2006) 103.

[107] T. Duguet, P. Bonche and P.-H. Heenen, Nucl. Phys. A 679 (2001) 427.

[108] Zhen-Hua Zhang, Jin-Yan Zeng, En-Guang Zhao and Shan-Gui Zhou, Phys. Rev. C 83 (2011) 011304(R).

[109] B. Nerlo-Pomorska, K. Pomorski and J. Bartel, Phys. Rev. C 84 (2011) 044310 .

[110] Yue Shi, J. Dobaczewski and P.T. Greenlees, Phys. Rev. C 89 (2014) 034309 .

[111] M. Bender, P. Bonche, T. Duguet and P.-H. Heenen, Nucl. Phys. A 723 (2003) 354 .

[112] F. Al-Khudair, Gui-Lu Long and Yang Sun, Phys. Rev. C 79 (2009) 034320 .

[113] J.L. Egido and L.M. Robledo, Phys. Rev. Lett. 85 (2000) 1198.

[114] H.L. Liu, F.R. Xu and P.M. Walker, Phys. Rev. C 86 (2012) 011301(R).

[115] Z.-H. Zhang, J. Meng, En-G. Zhao and S.-G. Zhou, Phys. Rev. C 87 (2013) 054308. 
[116] A.V. Afanasjev, H. Abusara, E. Litvinova and P. Ring, Jour. Phys.: Conf. Ser. 312 (2011) 092004.

[117] I. Hamamoto and H. Sagawa, Nucl. Phys. A 327 (1979) 99.

[118] W. Nazarewicz, J. Dudek, R. Bengtsson, and I. Ragnarsson, Nucl. Phys. A 435 (1985) 397.

[119] S. Ćwiok et al., Comput. Phys. Commun. 46 (1987) 379.

[120] A. Chatillon, PhD Thesis, Université Claude Bernard - Lyon I, 2005.

[121] S.P. Ivanova, A.L. Komov, L.A. Malov and V.G. Solov'ev, Sov. J. Part. Nucl. Phys. 7 (1976) 175.

[122] F.G. Kondev et al., Proceeding of the International Conference on Nuclear Data for Science and Technology 2007, EDP sciences.

[123] G.G. Adamian, N.V. Antonenko and W. Scheid, Phys. Rev. C 81 (2010) 024320 .

[124] R.V. Jolos, L.A. Malov, N.Yu. Shirikova and A.V. Sushkov, J. Phys. G: Nucl. Part. Phys. 38 (2011) 115103.

[125] H.L Liu, F.R. Xu, P.M. Walker and C.A. Bertulani, Phys. Rev. C 83 (2011) 011303(R).

[126] H.L Liu, P.M. Walker and F.R. Xu, Phys. Rev. C 89 (2014) 044304.

[127] A.V. Afanasjev and S. Shawaqfeh, Phys. Lett. B 706 (2011) 177.

[128] V.G. Soloviev and T. Siklos, Nucl. Phys. 59 (1964) 145.

[129] V.G. Solov'ev, A.V. Sushkhov and N.Yu. Shirikova, Sov. J. Nucl. Phys. 54 (1991) 748.

[130] J. Zhao, B.-N. Lu, E.-G. Zhao and S.-G. Zhou, Phys. Rev. C 86 (2012) 057304.

[131] Y.-S. Chen, Y. Sun and Z.-C Gao, Phys. Rev. C 77 (2008) 061305(R).

[132] M. Warda and J.L. Egido, Phys. Rev. C 86 (2012) 014322. 
[133] X.M. Fu, F.R. Xu, C.F. Jiao, W.Y. Liang, J.C. Pei and H.L. Liu, Phys. Rev. C 89 (2014) 054301.

[134] T. Bürvenich, K. Rutz, M. Bender, P.G. Reinhard, J.A. Maruhn and W. Greiner, Eur. Phys. J. A 3 (1998) 139.

[135] M. Kortelainen et al., Phys. Rev. C 85 (2015) 024304.

[136] Y. Hatsukawa et al., Nucl. Phys. A 500 (1989) 90.

[137] P.T. Greenlees et al., J. Phys. G: Nucl. Part. Phys. 24 (1998) L63.

[138] E.D. Donets, V.A. Shchegolev and V.A. Ermakov, Sov. J. Nucl. Phys. 2 (1966) 723.

[139] Y. Aritomo, Phys. Rev. C 75 (2007) 024602.

[140] V.Yu. Denisov, Prog. Part. Nucl. Phys. 46 (2001) 303.

[141] W.J. Świątecki, K. Siwek-Wilczyńska and J. Wilczyński, Phys. Rev. C $71(2005) 014602$.

[142] K. Siwek-Wilczyńska, I. Skwira-Chalot and J. Wilczyński, Int. J. Mod. Phys. E 16 (2007) 483.

[143] V. Zagrebaev and W. Greiner, Phys. Rev. C 78 (2008) 034610.

[144] G. Mandaglio et al., EPJ Web of conferences, 38 (2012) 01001.

[145] R.K. Choudhury and Y.K. Gupta, Phys. Lett. B 731 (2014) 168.

[146] T. Cap, K. Siwek-Wilczyńska and J. Wilczyński, Phys. Lett. B 736 (2014) 478.

[147] Yu.Ts. Oganessian et al., Phys. Rev. C 79 (2009) 024608.

[148] B. Avez et al., ArXiv:1111.2913v3 [nucl-ex].

[149] M.T. Magda and J.D. Leyba, Int. J. Mod. Phys. E 1 (1992) 221.

[150] D. Lee et al., Phys. Rev. C 27 (1983) 2656.

[151] V.I. Zagrebaev and W. Greiner, Phys. Rev. C 87 (2013) 034608. 
[152] C. Simenel, Eur. Phys. J. A 48 (2012) 152.

[153] W. Loveland, A.M. Vinodkumar, D. Peterson and J.P. Greene, Phys. Rev. C 83 (2011) 044610.

[154] A. Vogt et al., to be published.

[155] A. Lopez-Martens et al., Nucl. Phys. A 852 (2011) 15.

[156] J.S. Berryman et al., Phys. Rev. C 81 (2010) 064325.

[157] J. Rissanen et al., Phys. Rev. C 88 (2013) 044313.

[158] W. Loveland, Phys. Rev. C 76 (2007) 014612

[159] W. Loveland, Proceedings of the Fifth International Conference on ICFN5. Edited by Hamilton and Ramayya. Published by World Scientific Publishing, pp. 295-302.

[160] S. Akkoyun et al., Nucl. Instr. Meth. A 668 (2012) 26.

[161] S. Paschalis et al., Nucl. Instr. Meth. A 709 (2013) 44.

[162] D. Seweryniak, FMA and AGFA collaborations, Nucl. Instr. Meth. B 317 (2013) 274.

[163] C. Schmitt et al., Nucl. Instr. Meth. A 621 (2010) 558.

[164] A. Maj et al., Acta Phys. Pol. B 40 (2009) 565.

[165] Ch. Theisen et al., Nucl. Instr. Meth. A 747 (2014) 69.

[166] N. Madhavan et al., Pramana J. Phys. 75 (2010) 317.

[167] N. Madhavan et al., EPJ Web of Conf. 17 (2011) 14003.

[168] G. Mohanto et al., Phys. Rev. C 88 (2013) 034606.

[169] S. Muralithar et al., Nucl. Instr. Meth. A 662 (2010) 281.

[170] E.G. Jackson et al., Nucl. Instr. Meth. A 774 (2015) 34.

[171] E.G. Jackson, PhD Thesis, University of Massachusetts Lowell, 2015.

[172] H.W. Wilschut and V.L. Kravchuk, Nucl. Phys. A 734 (2004) 156.

[173] M.O. Frégeau et al., Phys. Rev. Lett. 108 (2012) 122701. 